The Astrophysical Journal, 684:136-152, 2008 September 1

(C) 2008. The American Astronomical Society. All rights reserved. Printed in U.S.A.

\title{
THE CONTRIBUTION OF AGNs AND STAR-FORMING GALAXIES TO THE MID-INFRARED AS REVEALED BY THEIR SPECTRAL ENERGY DISTRIBUTIONS
}

\author{
C. Gruppioni,${ }^{1}$ F. Pozzi,${ }^{2}$ M. Polletta,${ }^{3}$ G. Zamorani,${ }^{1}$ F. La Franca,${ }^{4}$ N. Sacchi,${ }^{4}$ A. Comastri,${ }^{1}$ \\ L. Pozzetti, ${ }^{1}$ C. Vignali,${ }^{2}$ C. Lonsdale, ${ }^{5}$ M. Rowan-Robinson ${ }^{6}{ }^{\text {J. Surace }},{ }^{5}$ \\ D. Shupe, ${ }^{5}$ F. Fang, ${ }^{5}$ I. Matute, ${ }^{7}$ and S. Berta ${ }^{8}$ \\ Received 2007 June 29; accepted 2008 April 25
}

\begin{abstract}
We present the broadband SEDs of the largest available highly complete (72\%) spectroscopic sample of MIRselected galaxies and AGNs at intermediate redshift. The sample contains 203 extragalactic sources from the $15 \mu \mathrm{m}$ ELAIS-SWIRE survey, all with measured spectroscopic redshift. Most of these sources have full multiwavelength coverage from the FUV (GALEX) to the FIR (Spitzer) and lie in the redshift range $0.1<z<1.3$. This large sample allows us for the first time to characterize the spectral properties of sources responsible for the strong evolution observed in the MIR. Based on SED-fitting, we have classified the MIR sources, identifying AGN signatures in about $50 \%$ of them. This fraction is significantly higher than that derived from optical spectroscopy $(\sim 29 \%)$ and is due in particular to the identification of AGN activity in objects spectroscopically classified as galaxies (the spectroscopic classification may be somewhat unreliable because of host galaxy dilution in the optical). It is likely that in most of our objects, the AGN is either obscured or low luminosity, and thus dominates the energetic output only in the MIR, showing up just in the range where the host galaxy SED has a minimum. The fraction of AGNs strongly depends on flux density, with that derived through the SED-fitting about $20 \%$ at $S_{15 \mu \mathrm{m}} \sim 0.5-1 \mathrm{mJy}$ and gradually increasing to $100 \%$ at $S_{15 \mu \mathrm{m}}>10 \mathrm{mJy}$, while that obtained from optical spectroscopy is never $>30 \%$, even at higher flux densities. Our results will be very useful for updating all models aimed at interpreting the deep IR survey data and in particular for constraining the nature and role of dust-obscured systems in the intermediate/high-redshift universe.
\end{abstract}

Subject headings: cosmology: observations — galaxies: evolution — galaxies: Seyfert — galaxies: starburst — infrared: galaxies

\section{INTRODUCTION}

Understanding the overall spectral energy distribution (SED) of sources responsible for the observed cosmic infrared background (CIRB) is a crucial tool for unveiling the nature and evolution of galaxies and AGNs in the infrared (IR) and for obtaining a complete picture of the history of star formation and obscured AGN activity in the universe. The mid- and far-infrared (MIR and FIR) regions of the electromagnetic spectrum efficiently probe the population of actively star-forming galaxies and dust-obscured AGNs.

The Infrared Astronomical Satellite (IRAS) has sampled the local universe $(z<0.2)$ in the MIR/FIR band, discovering ultraluminous infrared galaxies (ULIRGs: $L_{\mathrm{IR}}>10^{12} L_{\odot}$; Sanders \& Mirabel 1996; Lonsdale et al. 2006) and first showing some evidence for their strong evolution (i.e., Hacking et al. 1987; Lonsdale \& Hacking 1989; Saunders et al. 1990).

\footnotetext{
1 INAF-Osservatorio Astronomico di Bologna, via Ranzani 1, I-40127 Bologna, Italy.

2 Dipartimento di Astronomia, Università di Bologna, via Ranzani 1, I-40127 Bologna, Italy.

3 Institut d'Astrophysique de Paris, 98 bis Boulevard Arago, 75014 Paris, France.

4 Dipartimento di Fisica, Università degli Studi “Roma Tre," via della Vasca Navale 84, I-00146 Rome, Italy.

5 Infrared Processing and Analysis Center, Mail Stop 100-22, California Institute of Technology, Pasadena, CA 91125.

6 Astrophysics Group, Blackett Laboratory, Imperial College of Science Technology and Medicine, Prince Consort Road, London SW7 2BZ, UK.

7 INAF-Osservatorio Astrofisico di Arcetri, Largo E. Fermi 5, I-50125 Florence, Italy.

${ }^{8}$ Dipartimento di Astronomia, Universitá di Padova, vicolo Osservatorio 2, I-35122 Padua, Italy.
}

Extragalactic source counts from different surveys over a wide flux range obtained with the ISOCAM instrument (Cesarsky et al. 1996) on board the Infrared Space Observatory (ISO; Kessler et al. 1996) indicate that IR sources less extreme than ULIRGs (i.e., luminous infrared galaxies [LIRGs]: $L_{\mathrm{IR}}>10^{11} L_{\odot}$ ) have also evolved rapidly from $z \sim 0$ up to 1.2 , significantly faster than the rate deduced from optical surveys (i.e., Elbaz et al. 1999; Gruppioni et al. 2002; Pozzi et al. 2004).

The Spitzer Space Telescope (Werner et al. 2004) is now providing new insights into the IR population contributing to the CIRB, in particular in the MIPS $24 \mu \mathrm{m}$ band, where extragalactic source counts (Marleau et al. 2004; Papovich et al. 2004) have confirmed the existence of the rapidly evolving dust-obscured population discovered by ISOCAM, detecting the higher $z$ analogs $(1<z<3)$ of the ISOCAM galaxies. In particular, the deeper MIPS surveys are now able to resolve about $70 \%$ of the CIRB at $24 \mu \mathrm{m}$, with the bulk of this background originating in discrete sources at $z \lesssim 2$ (e.g., Papovich et al. 2004).

These results have stimulated the development of several evolutionary models for IR galaxies (i.e., Rowan-Robinson 2001; Franceschini et al. 2001; Chary \& Elbaz 2001; Xu et al. 2003; Lagache et al. 2003, 2004; Pozzi et al. 2004; Gruppioni et al. 2005; Pearson 2005), aimed at reproducing the MIR source counts and the observed redshift distributions. All these models are more or less able to reproduce the observed ISOCAM $15 \mu \mathrm{m}$ source counts, but none of them can provide an acceptable fit to the MIPS $24 \mu \mathrm{m}$ counts without any ad hoc changes. In particular, they tend to predict the characteristic turnover of the differential $24 \mu \mathrm{m}$ number counts at fluxes higher than observed. Even worse is the situation regarding the observed redshift distributions, both for ISOCAM and MIPS sources. Le Floc'h et al. (2005) and Pèrez-Gonzàlez et al. (2005), by studying the luminosity evolution 
of a sample of MIPS $24 \mu \mathrm{m}$ sources in the Chandra Deep FieldSouth (CDF-S) up to $z=1$ and in the range $1<z<3$, respectively, find that all the models considered in these works (Lagache et al. 2004; Chary et al. 2004; Gruppioni et al. 2005; Pearson $2005)$ fail in reproducing the differential counts at $24 \mu \mathrm{m}$ in different redshift slices and the total $z$-distribution. We must note that most of the redshifts considered in these works are photometric, and the analyzed fields are relatively small and can therefore be affected by cosmic variance. However, there seems to be a significant disagreement between all the evolutionary models existing in literature and the recent Spitzer data. Two main weaknesses are common to all these models:

1. They are based on very uncertain assumptions about the shape of the SED of MIR sources. In particular, they generally assume an invariant PAH emission and extrapolate a few local SEDs to high redshifts and luminosities $(L)$, either by keeping them fixed or artificially increasing their activity with luminosity (e.g., Chary \& Elbaz 2001).

2. They severely underestimate, or even completely neglect (e.g., Lagache et al. 2003; Chary \& Elbaz 2001), the AGN contribution in MIR sources (see Brand et al. 2006; Gruppioni et al. 2005). In fact, without either MIR spectroscopic data or a complete SED characterization, it is very difficult to disentangle starforming galaxies from AGNs (Genzel et al. 1998; Sajina et al. 2007). This is particularly true in cases in which spectroscopic classification based on optical line diagnostics is impossible, due to the absence of some of the required emission lines in the observed spectral range, to the significant differential line extinction produced by dust, or to the lack of spectroscopic data and deep $\mathrm{X}$-ray coverage. Since different evolutionary models are applied to the two populations, the misidentification of AGNs and starforming galaxies introduces large uncertainties in the predicted source counts and backgrounds.

The crucial wavelength range $(3-200 \mu \mathrm{m})$ covered by Spitzer with unprecedented sensitivity offers the opportunity to characterize for the first time the MIR/FIR spectral properties of a large number of sources over a significant extent in redshift and to study their evolution with $z$ and/or $L$, testing the model assumptions. In particular, thanks also to the extensive multiwavelength coverage available in several areas of the sky, it is now possible to study the broadband (from UV to FIR) spectral properties of the same sources responsible for the observed evolution in the MIR, thus constructing observational templates libraries over a large range of wavelengths.

In the southern field of the European Large Area ISO Survey (ELAIS; Oliver et al. 2000; Rowan-Robinson et al. 2004), S1, an extensive multiwavelength follow-up campaign, has been undertaken in recent years. This field, initially selected for ISO observations at 7,15 , and $90 \mu \mathrm{m}$, is now one of the six areas covered by the Spitzer Wide-Area Infrared Extragalactic Survey (SWIRE; Lonsdale et al. 2003) in all the IRAC and MIPS bands. In addition to ISO and Spitzer observations, the whole S1 field $\left(\sim 4 \mathrm{deg}^{2}\right)$ has been deeply surveyed in the radio (ATCA ; Gruppioni et al. 1999; Middleberg et al. 2008), in the optical ( $R$ band, ESO $3.6 \mathrm{~m}$; La Franca et al. 2004), and in the near-UV (NUV) and far-UV (FUV; Galaxy Evolution Explorer [GALEX]; Burgarella et al. 2005). The central square degree of S1 is covered by NIR (ESO SofI; Dias et al. 2008) and optical (ESO WFI; Berta et al. 2006) data, while $\sim 0.6 \mathrm{deg}^{2}$ have been observed in the X-ray (BeppoSAX, Alexander et al. 2001; XMM-Newton, Puccetti et al. 2006). Optical spectroscopic data are available for $\sim 20015 \mu \mathrm{m}$ ISOCAM sources (ESO $3.6 \mathrm{~m}$; La Franca et al. 2004) and for most of the XMM-Newton and some of the SWIRE $24 \mu \mathrm{m}$ sources with optical counterparts brighter than $R \approx 24$ (with ESO VIMOS; C. Feruglio et al. 2008, in preparation; N. Sacchi et al. 2008, in preparation).

Due to its extensive multiwavelength coverage and its large area extent, $\mathrm{S} 1$ is well suited to study in detail the broadband SEDs of a statistically significant sample of infrared galaxies and AGNs at intermediate redshifts $(z<1.5)$. To this end, we have selected the 203 extragalactic sources detected by ISOCAM at $15 \mu \mathrm{m}(S>0.5 \mathrm{mJy})$ in the S1 field (Lari et al. 2001) with $R<23$ and spectroscopic information available (La Franca et al. 2004, 2007). The same sample has previously been used to derive the first $15 \mu \mathrm{m}$ luminosity function of galaxies (Pozzi et al. 2004) and AGNs (Matute et al. 2002, 2006). The treatment of the infrared/ optical incompleteness of this sample for statistical purposes is described in detail by Gruppioni et al. (2002) and La Franca et al. (2004). The sample discussed here can be considered to be the largest available spectroscopic sample of IR galaxies and AGNs at intermediate redshift with such a high level of completeness (72\%), allowing us for the first time to characterize the spectral properties of the sources responsible for the strong evolution observed in the MIR. Although large spectroscopic samples are available in fields observed by Spitzer, such as the GOODS-CDF-S (see Vanzella et al. 2005, 2006), the targets are not selected to be MIR sources, but optical/NIR objects responding to given color criteria and likely to be at high redshifts. In the very near future, large spectroscopic samples of MIR- $(24 \mu \mathrm{m}-)$ selected sources, either locally (i.e., in the First Look Survey [FLS] by Marleau et al. 2007) or at high redshift (i.e., in the COSMOS area) will be made available to the community, allowing studies similar to the one presented here. At present, however, our sample is unique in pursuing this purpose. Indeed, most of our objects are LIRGs, with MIR fluxes in the crucial range between the IRAS surveys (200 mJy) and the Deep ISOCAM Surveys ( $0.1 \mathrm{mJy}$; Elbaz et al. 1999), where MIR source counts start diverging from no-evolution expectations. Most of these objects (except for type 1 AGNs) are in the redshift range $0.1<z<1.0$. We make use of all the available data, from FUV to FIR, to derive the SEDs of these sources and construct the first observational library of templates for MIR galaxies and AGNs at intermediate $z$. To interpret the observed SEDs, we perform a fit with several local template SEDs, representative of different classes of IR galaxies and AGNs (Polletta et al. 2007), comparing the resulting SED classification with the spectroscopic one.

The present paper is structured as follows. In $\S 2$ we describe the reference sample. In $\S 3$ we present the multiwavelength data set and the observed SEDs, discussing the SED-fitting procedure and results. In $\S 4$ we compare the original spectroscopic classification with the SED classification. In $\S 5$ we present the revised $15 \mu \mathrm{m}$ counts for AGNs and galaxies, and we discuss the results and present our conclusions.

Throughout the paper we adopt a cosmological model with $\Omega_{m}=0.3, \Omega_{\Lambda}=0.7$, and $H_{0}=70 \mathrm{~km} \mathrm{~s}^{-1} \mathrm{Mpc}^{-1}$. The magnitudes discussed here are in the Vega system.

\section{THE SAMPLE}

Our reference sample is the Lari et al. (2001) catalog of 462 sources selected at $15 \mu \mathrm{m}$ (LW3 band) in the ELAIS southern field S1 [centered at $\alpha(\mathrm{J} 2000.0)=00^{\mathrm{h}} 34^{\mathrm{m}} 44.4^{\mathrm{s}}, \delta(\mathrm{J} 2000.0)=$ $-43^{\circ} 28^{\prime} 12^{\prime \prime}$ and covering about $\left.2^{\circ} \times 2^{\circ}\right]$. This sample, complete at the $5 \sigma$ level, is the only ISOCAM sample covering the whole flux density range $0.5-150 \mathrm{mJy}$, thus linking IRAS to the Deep ISOCAM Surveys (Elbaz et al. 1999). The source counts at $15 \mu \mathrm{m}$ obtained from that catalog sample the flux density region where observed counts start diverging from no-evolution models, as discussed by Gruppioni et al. (2002). La Franca et al. (2004) presented 
$R$-band data for a highly reliable subsample of 406 out of a total of $46215 \mu \mathrm{m}$ sources of the Lari et al. (2001) catalog. The $R$-band data were obtained by an ESO imaging campaign with the DFOSC instrument mounted on the $1.5 \mathrm{~m}$ ESO Danish telescope at La Silla (Chile), providing a reliable optical counterpart down to $R \sim 23$ for 317 of them, thus reaching a $95 \%$ completeness level. Spectroscopic observations of the optical counterparts of the ISOCAM S1 sources were carried out at the AAT 2dF, ESO Danish 1.5 and $3.6 \mathrm{~m}$ telescopes, and the New Technology Telescope (NTT) ( $\mathrm{La}$ Franca et al. 2004), providing a secure spectroscopic identification for 290 ISOCAM sources (72\% of the whole highly reliable ELAIS-S1 sample). La Franca et al. (2004) have classified 199 of the spectroscopically identified sources as extragalactic objects (25 type 1 AGNs, 23 type 2 AGNs, 9 LINERs, 32 starburst galaxies, $100 \mathrm{H} \alpha$-emitter galaxies, 3 early-type galaxies, and 7 unclassified but with measured redshift), the remaining 91 as stars. Details about the identification completeness of the extragalactic sample for statistical uses are given in La Franca et al. (2004). These 199 extragalactic objects with measured redshift are the same objects previously used for the statistical analysis of the evolution of galaxies and AGNs by Pozzi et al. (2004) and Matute et al. (2002, 2006), respectively. With respect to the La Franca et al. (2004) catalog, two more $15 \mu \mathrm{m}$ sources were spectroscopically identified through Very Large Telescope (VLT) VIMOS observations (La Franca et al. 2007): ELAISC15_J003317-431706 is a $R=24.3$ galaxy showing [O II] emission at redshift 0.689 , while ELAISC15_J003447-432447 is an AGN2 at redshift 1.076. Moreover, we derived redshifts for two additional $15 \mu \mathrm{m}$ sources: for ELAISC15_J003915-430426 we found a $z$-value of 0.013 in the NED database, while for ELAISC15_J003545-431833 we were able to measure $z$ through a more accurate reduction of the spectrum. Three sources with previously poor-quality spectra had their spectroscopic classification changed after being reobserved with VIMOS at ESO VLT (La Franca et al. 2007): ELAISC15 J003330-431553, which was wrongly classified as a starburst galaxy at $z=0.473$, showed broad C III and Mg II emission at $z=$ 2.170, typical of AGN1 activity; ELAISC15_J003603-433155, which was classified as AGN2, showed a broad Mg II emission typical of AGN1 activity; and ELAISC15_J003622-432826, which was classified as a starburst galaxy, showed a clear [O III]/ $\mathrm{H} \beta$ ratio typical of AGN2 activity.

In summary, we have considered 203 extragalactic sources selected at $15 \mu \mathrm{m}$ with $R<23$, spectroscopic redshift and multiband photometry.

\section{THE SEDS}

\subsection{The Multiwavelength Data Set}

The objects we focus on cover the redshift range 0.01-3.09 (0.01-1.30, excluding type $1 \mathrm{AGNs}$ ) and more than 2 orders of magnitude in $15 \mu \mathrm{m}$ flux (0.5-60 mJy). The multiband SEDs for these sources have been constructed by looking for counterparts in all the multiwavelength catalogs from FUV to FIR available in the ELAIS-S1 field. Given for granted the optical $R$-bandspectroscopic association performed by La Franca et al. (2004), we have searched for associations in the GALEX Deep Imaging Survey (DIS), ESO Spitzer Imaging Extragalactic Survey (ESIS), $J, K_{S}$, and SWIRE catalogs. The cross-correlation radii between the $15 \mu \mathrm{m}$ or optical positions and the different matching catalogs positions have been chosen equal to the value above which spurious associations start dominating (i.e., Hook et al. 1998).

The ultraviolet Galaxy Evolution Explorer (GALEX; Martin et al. 2005) DIS is observing $\sim 100 \mathrm{deg}^{2}$ in 12 different areas of the sky, including ELAIS-S1 (Burgarella et al. 2005). Twelve
ELAIS-S1 GALEX tiles are already available to the public as part of the second data release (GR2). The exposure times for these tiles vary from $\sim 3000$ to $\sim 50,000 \mathrm{~s}$. The photometric catalogs in the FUV (1530 $\AA)$ and NUV (2310 $\AA$ ) have been crosscorrelated with the optical positions of the ISOCAM source counterparts, with a matching radius of $3^{\prime \prime}$. Then all the possible UV counterparts have been visually inspected on the GALEX images, in order to reject spurious associations. We found 176 likely associations with the NUV catalog, 169 of which are also in the FUV one.

ESIS (Berta et al. 2006) has covered the central $1.5 \mathrm{deg}^{2}$ of ELAIS-S1 with WFI $2.2 \mathrm{~m}$ deep observations in the $B, V$, and $R$ bands, reaching $95 \%$ completeness at $B, V \sim 25$, and $R \sim 24.5$. The cross-correlation between the original optical positions and the ESIS positions (within 2") have produced a good match for 90 sources (those falling in the area covered by ESIS), thus providing additional optical $(B, V)$ magnitudes for about $45 \%$ of the 203 ISOCAM sources. In the area not covered by ESIS, $R$ magnitudes from the La Franca et al. (2004) catalog and $b_{J}$ magnitudes from the APM survey ( $\sim 90 \%$ complete to $b_{J}=20.5$; Maddox et al. 1990) have been used.

The central square degree of the ELAIS-S1 area (S1-5) was the target of NIR $J$ and $K_{S}$ imaging with NTT SOFI, reaching $J \simeq 21$ and $K_{s} \simeq 20$ (Vega) $^{9}$ (I. Matute et al. 2008, in preparation). The optical positions of the $15 \mu \mathrm{m}$ source counterparts have been cross-correlated with those from the NIR catalog, using a matching radius of $2^{\prime \prime}$. Thirty unique NIR counterparts have been found in the central square degree of ELAIS-S1, while additional NIR data from the Two Micron All Sky Survey (2MASS; Jarrett et al. 2000) are available over the whole $I S O$ area, providing 110 matches (10 of which are in common with the S1- $K_{S}$ ones). In total we found $K_{s}$-band magnitudes for 130 and $J$-band magnitudes for 30 out of the $20315 \mu \mathrm{m}$ sources.

The Spitzer SWIRE observations in ELAIS-S1 cover the whole ISO region and a total sky area of $\sim 7 \mathrm{deg}^{2}$ in all the IRAC and MIPS bands, reaching $5 \sigma$ sensitivities of $4.1,8.5,48.2,53.0$, $256.0,26 \times 10^{3}$ and $166 \times 10^{3} \mu \mathrm{Jy}$ in the $3.6,4.5,5.8,8.0,24$, 70 , and $160 \mu \mathrm{m}$ channels, respectively (Lonsdale et al. 2004). The SWIRE data in ELAIS-S1 were released to the community in the autumn of 2005 (Data Release 3 [DR3]) through the querybuilding Gator at the NASA/Infrared Science Archive. ${ }^{10}$ However, for purposes of this work, we had access to the SWIRE working catalogs, which contain all of the sources in the public catalog but reach deeper flux densities. Details about the SWIRE data reduction, carried out by the Spitzer Science Center and SWIRE team, can be found in the Data Release paper (Surace et al. 2005). ${ }^{11}$ For the IRAC fluxes and positions we used the bandmerged working catalog, consisting of IRAC and MIPS $24 \mu \mathrm{m}$ fluxes associated with each other. While the public catalog is based on $3.6 \mu \mathrm{m}$, requiring detections at 3.6 and $4.5 \mu \mathrm{m}$ above specific signal-to-noise ratio (SN) thresholds (10 at $3.6 \mu \mathrm{m}$ and 5 at $4.5 \mu \mathrm{m}$ ), the working catalog contains all detections, at any $\mathrm{S} / \mathrm{N}$, even if a source is detected only in one IRAC band. For the 24,70 , and $160 \mu \mathrm{m}$ fluxes and positions, we used the single-band-only catalogs, which contain all the sources detected only in the MIPS waveband in question. The IRAC positional accuracy is about $0.2^{\prime \prime}-0.4^{\prime \prime}$ in all bands, while the MIPS ones are $\sim 1^{\prime \prime}, 4^{\prime \prime}$, and $8^{\prime \prime}$ at 24,70 , and $160 \mu \mathrm{m}$, respectively. Fixed aperture photometry is used for pointlike objects, corrected for aperture losses (we have

\footnotetext{
9 As part of the ESO Large Program 170.A-0143, PI: A. Cimatti.

10 At http://irsa.ipac.caltech.edu/applications/Gator.

11 Also http://data.spitzer.caltech.edu/popular/swire/20050603_enhanced_v1/ Documents/SWIRE2_doc_083105.pdf.
} 
considered the SWIRE aperture2 (1.9" in radius) for both IRAC and MIPS), while ad hoc aperture photometry (i.e., as large as needed to recover the whole flux of very extended sources) has been performed by us directly on images for the extended sources, for which even the SExtractor's Kron fluxes (those suggested for extended sources) are found to underestimate the total flux densities (up to a factor of $\sim 2$ at $24 \mu \mathrm{m}$ ). For the match with the IRAC band-merged catalog we have considered the optical positions, searching for counterparts within a radius of $3^{\prime \prime}$, while for match with the MIPS catalogs we have considered the ISOCAM positions with searching radii of $5^{\prime \prime}, 10^{\prime \prime}$, and $20^{\prime \prime}$ at 24,70 , and $160 \mu \mathrm{m}$, respectively. All but one of the $20315 \mu \mathrm{m}$ sources are detected in at least two IRAC bands (most in all four bands), while 200 are detected at 24,110 at 70 , and 59 at $160 \mu \mathrm{m}$. The matches with 24,70 , and $160 \mu \mathrm{m}$ sources have been visually inspected to be sure they were not mismatches. From the visual inspection of the MIPS images we have found clear detections for 43 and 45 additional sources at 70 and $160 \mu \mathrm{m}$, respectively. However, we have chosen not to derive flux densities for these sources, considering in this work only those included in the SWIRE catalogs.

In Table 1 we report the multiwavelength informations available for all the $20315 \mu \mathrm{m}$ sources. The source name from the Lari et al. (2001) catalog is reported in the first column, while the flux density (in $\mu \mathrm{Jy}$ ) in all bands (from FUV to FIR), the redshift and the total infrared luminosity (obtained by integrating the bestfitting SED in the range 8-1000 $\mu \mathrm{m}$ ) are reported in the following columns. In the last two columns the spectroscopic and SEDfitting classifications (see $\S 4$ ) are presented. In cases of no detection, we have quoted a $3 \sigma$ upper limit, while ellipses indicate no data coverage.

\subsection{The Template Library}

We have made use of all the available data, from FUV to FIR, to derive the SEDs of these 203 sources and construct the first highly complete observational sample of SEDs for MIR galaxies and AGNs at intermediate $z$ to be used for statistical purposes (i.e., luminosity function). To interpret and classify the observed SEDs, we have performed a fit with several local template SEDs, representative of different classes of IR galaxies and AGNs (including three ellipticals of different ages, one lenticular, seven spirals, three starbursts, three QSOs, one reddened QSO, Seyfert 1, 1.8, and 2, and two ULIRGs, containing both a starburst and an AGN component) in the wavelength range between 0.1 and $1000 \mu \mathrm{m}$ (Polletta et al. 2007), appropriately redshifted to the rest frame. The full library of galaxy and AGN templates (excluding the three ellipticals, since none of our sources could be fitted by any of them) is shown in Figure 1. The elliptical, spiral, and starburst templates were generated with the GRASIL code (Silva et al. 1998). The spiral templates range from early to late types ( $\mathrm{S} 0-\mathrm{Sdm})$, the starburst templates correspond to the SEDs of NGC 6090, M82, and Arp 220. Templates of moderately luminous AGNs, representing Seyfert 1.8 and Seyfert 2 galaxies, were obtained by combining models and data from a large sample of Seyfert galaxies. The AGN templates include three templates representing optically selected QSOs with different values of IR/optical flux ratios (QSO, QSO_high, and QSO_low) and one reddened QSO (red_QSO). The composite $(\mathrm{AGN}+\mathrm{SB})$ templates are empirical templates created to fit the SEDs of the heavily obscured BAL QSO Mrk 231 (Berta 2005) and the Seyfert 2 galaxy IRAS 19254-7245 South (Berta et al. 2003). These objects contain a powerful starburst component, mainly responsible for their FIR emission, and an AGN component that contributes to the MIR (Farrah et al. 2003). With respect to existing template libraries derived from empirical SEDs (Coleman et al. 1980) or from models (Bruzual \& Charlot 2003;
Fioc \& Rocca-Volmerange 1997; Silva et al. 1998; Devriendt et al. 1999), this library has a broader wavelength coverage and variety of spectral types. Examples of the application of this library to various types of SWIRE sources can be found in Lonsdale et al. (2004), Franceschini et al. (2005), Hatziminaoglou et al. (2005), Jarrett et al. (2006), Polletta et al. (2006, 2007), Weedman et al. (2006), and Tajer et al. (2007).

To avoid biasing the results in favor of a small subset of templates, we adopt the full library of Polletta et al. (2007) to fit the SEDs. Although some templates are obtained from modeling the SEDs of ultraluminous objects (ULIRGs; $L_{\mathrm{IR}}>10^{12} L_{\odot}$, e.g., Mrk 231, I19254, and Arp 220), they are kept in the library, since they well represent a large fraction of the Spitzer population at several flux and luminosity levels (see, e.g., Franceschini et al. 2005; Polletta et al. 2006, 2007; Alonso-Herrero et al. 2006).

\subsection{The Fitting Method}

The SEDs of our sources are fitted using an automated fitting routine contained in the Photometric Analysis for Redshift Estimations (LE PHARE) software by Arnouts \& Ilbert, ${ }^{12}$ offering the possibility of using any template library and filters set. LE PHARE (Ilbert et al. 2006) is a publicly available set of FORTRAN programs aiming at computing photometric redshifts through the best-fitting SED analysis.

The program is based on a simple $\chi^{2}$-fitting method between the expected magnitudes and an observed photometric catalog.

Since we already know the spectroscopic redshift of our galaxies, we have fixed $z$ and have used LE PHARE only to obtain the best-fitting SED through a comparison with the template SEDs. No extinction was added to the templates, since some of them are already intrinsically extinct. Note that by using a spectroscopically complete sample we are able to avoid all the uncertainties due to the photometric redshift measurements, which greatly increase the degeneracy in the SED-fitting procedure. Moreover, for the first time we can compare the results from optical spectroscopy with the broadband SED shape of a statistically significant sample of MIR galaxies and AGNs.

We make the basic assumption that the SED shapes seen at low redshifts can also well represent the higher redshift objects. At the end of our procedure we would be able to verify this assumption. The template library used to fit our data contains a finite number of SEDs (21), representative of given classes of local infrared objects, which do not vary with continuity from one class to another (there are large gaps in the parameter space). Therefore, the quality of the fit depends not only on the photometric errors, but also on the template SED uncertainties. For this reason, in our fitting procedure, in addition to the photometric errors on data, we need to take into account also the uncertainties due to the template SEDs "discretization." To estimate and consider the total uncertainty on both the photometric data and the template SEDs, we have proceeded as follows. First, we have increased all the formal catalog errors (probably too small) by a reasonable given amount, ranging from 5\% in the UV and optical bands up to $\sim 15 \%$ in the MIPS bands. We have run LE PHARE on all the sources, obtaining a first "estimate" of the best-fitting SED classification. We have then computed the distributions of the $\left(S_{\text {object }}-\right.$ $\left.S_{\text {template }}\right)_{\text {band }} /\left(\sigma_{\text {object }}\right)_{\text {band }}$ values in each of the considered photometric band (where $S_{\text {object }}$ and $\sigma_{\text {object }}$ are the flux density and the relative error of the source, and $S_{\text {template }}$ the flux of the template in the considered band), iteratively increasing the photometric errors until we have obtained a Gaussian distribution with $\sigma \simeq 1$. This

12 Available at http://www.oamp.fr/people/arnouts/LE_PHARE.html. 


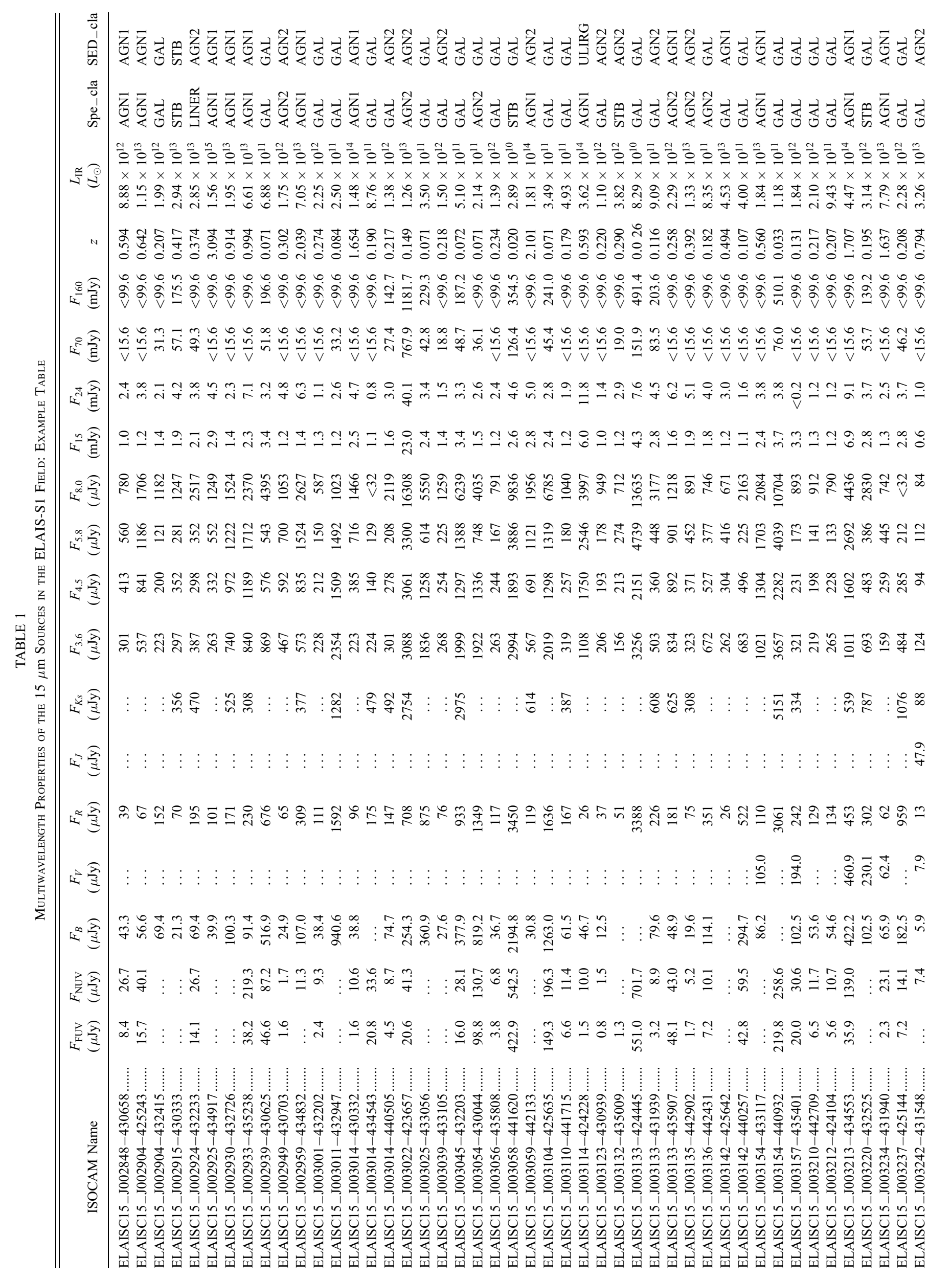




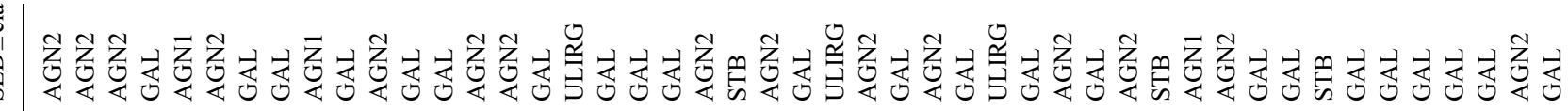

$\frac{\pi}{0}$

के

$\stackrel{ }{\exists}$

노욜

证礉

过畓

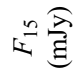

这要

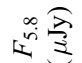

苛

(1)

哭要

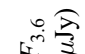

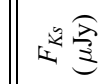

노올

또종

노 중

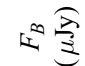

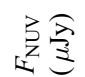

蛋䍃

芯

年

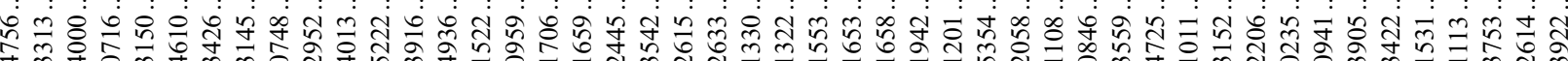

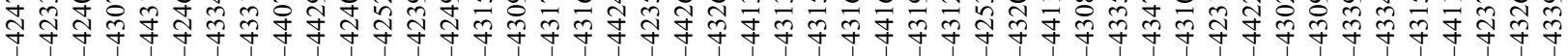

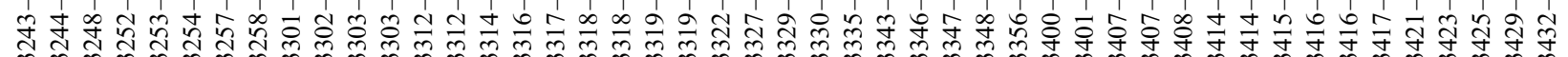

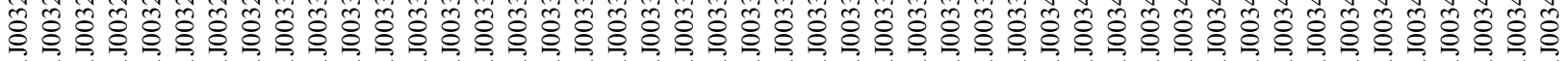

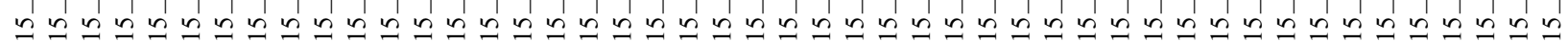

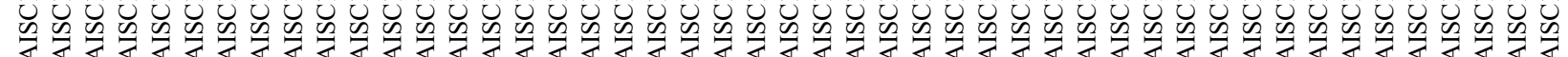

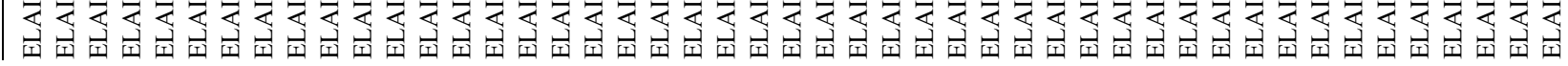




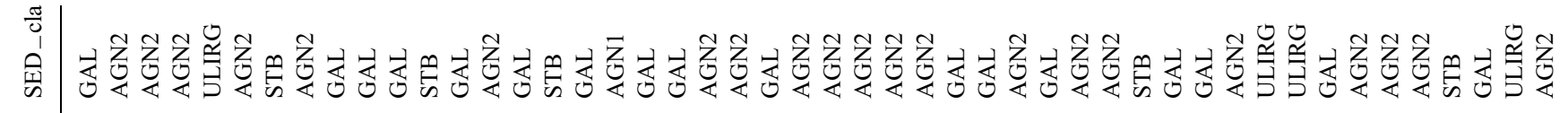

$\frac{\pi}{0}$

के

의

N

졸

졸

过尽

证态

这忟

$\infty \sqrt{-\infty}$

过资

둘

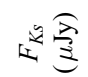

玨㢳

또용

노을

禹带

运罂

遍害

范

हี

$\sum$

○

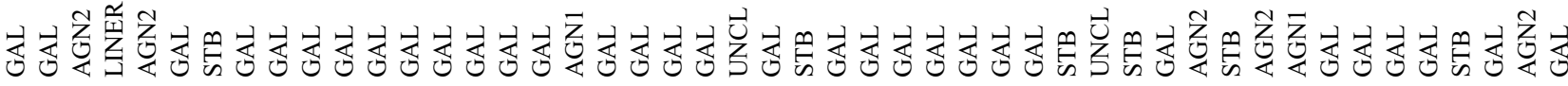

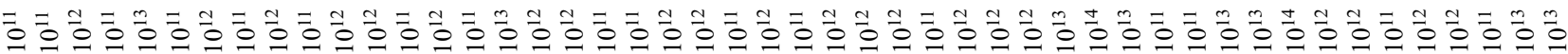

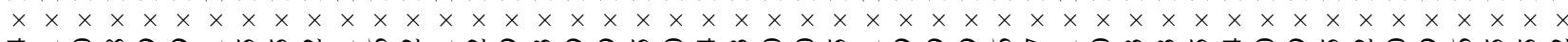

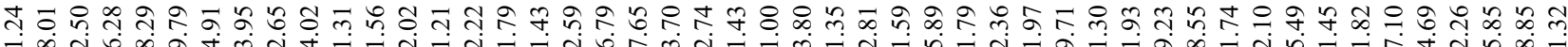

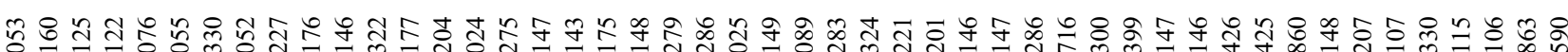

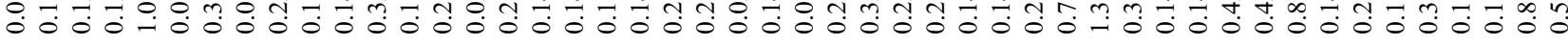

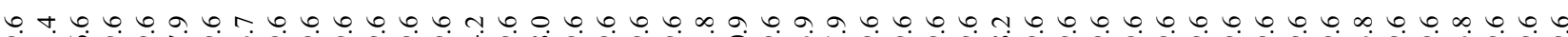

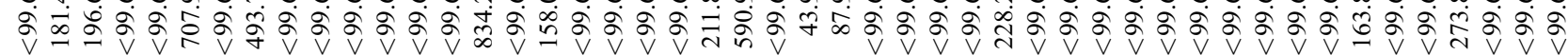

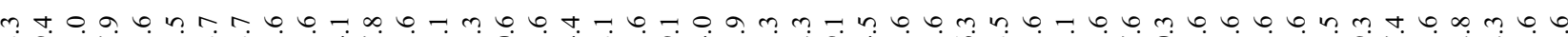

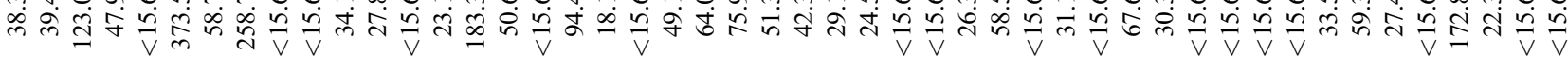

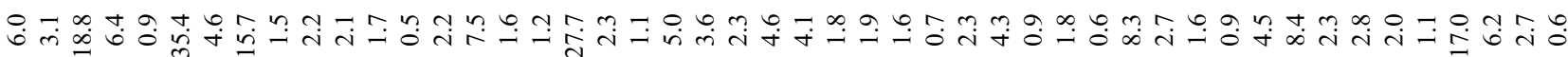

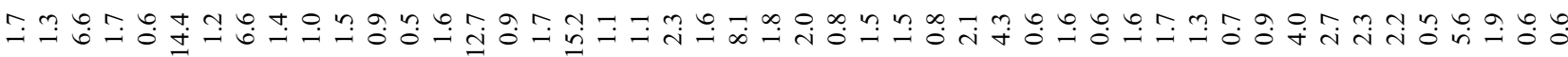

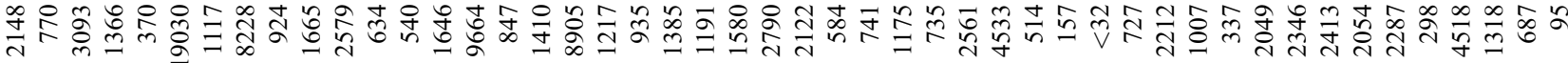

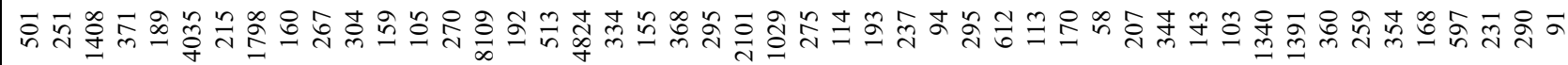

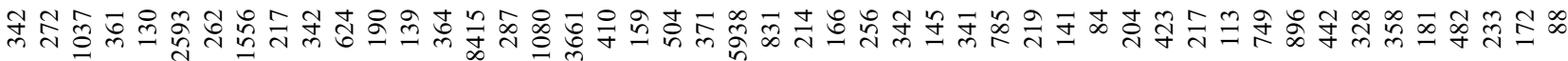

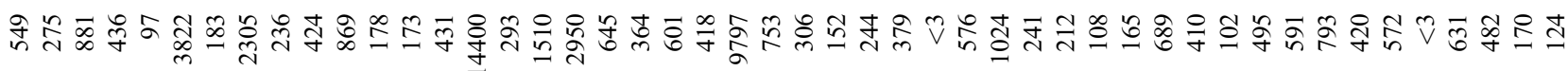

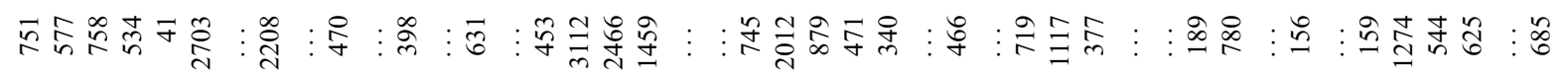
$\vdots:$
$: \stackrel{\circ}{\stackrel{a}{\vec{I}}}$

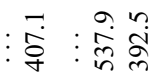

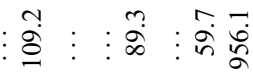

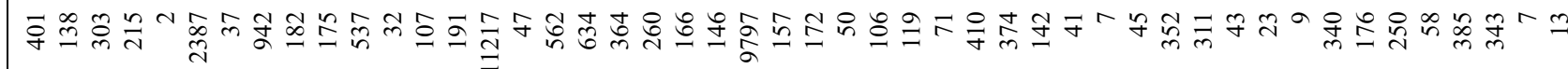

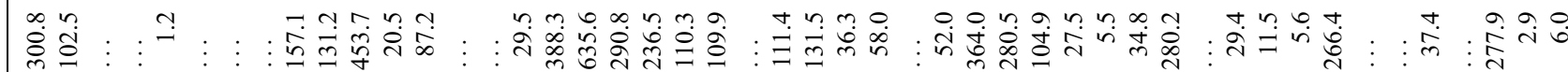

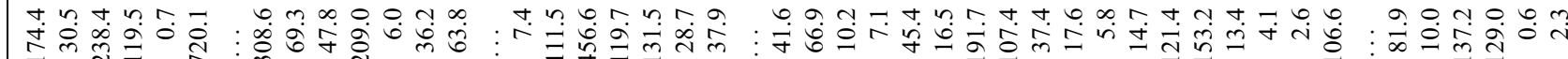

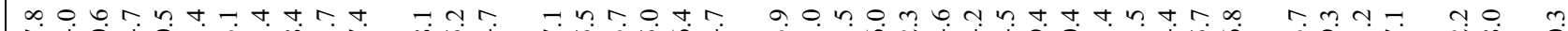

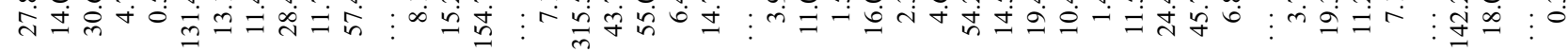

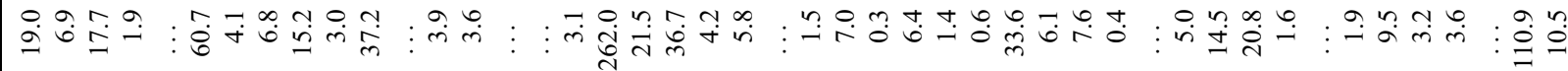

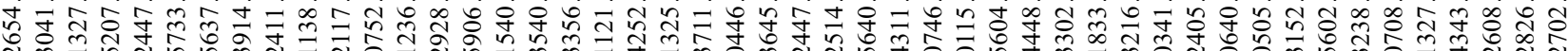

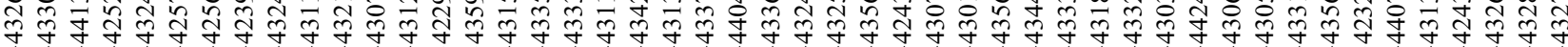

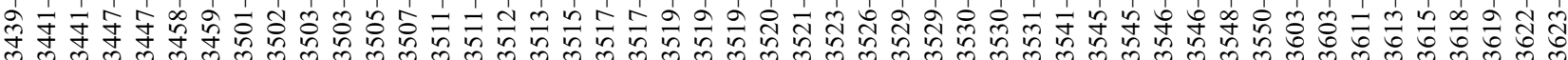

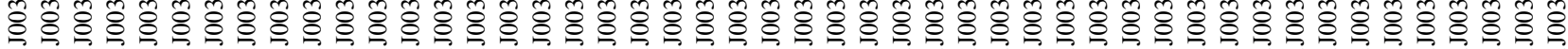

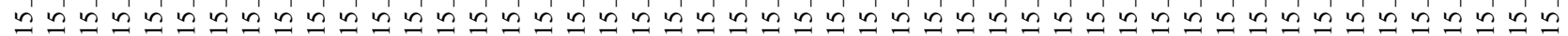

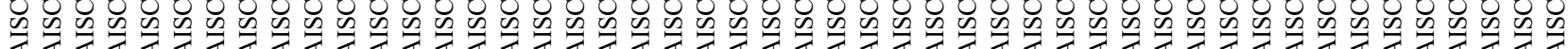

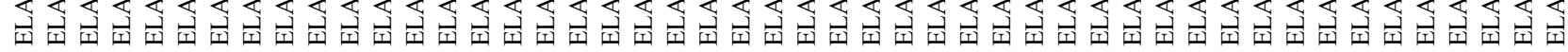




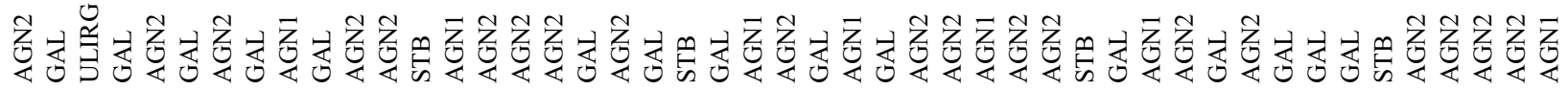

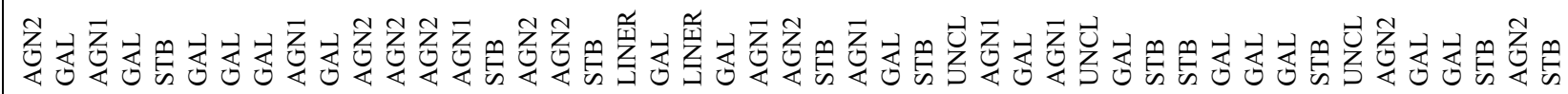

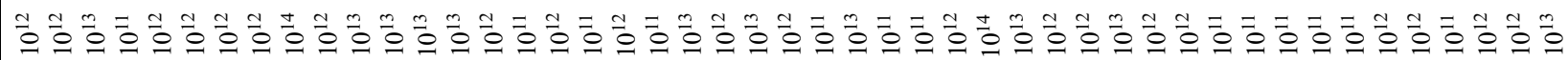

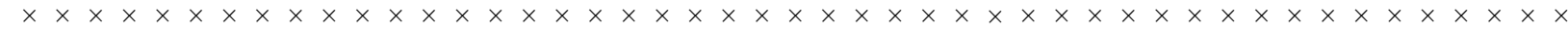

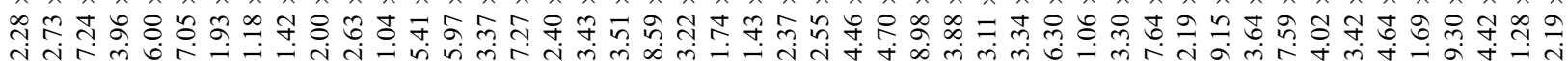

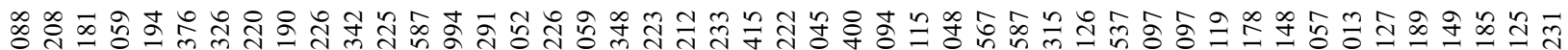

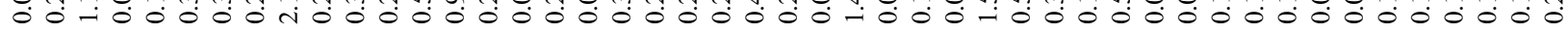

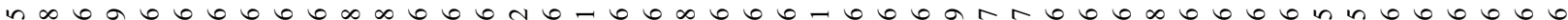

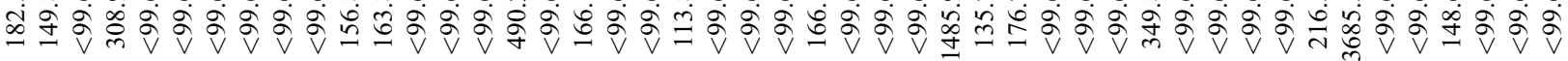

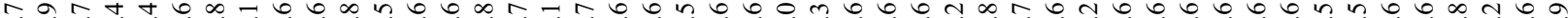
要

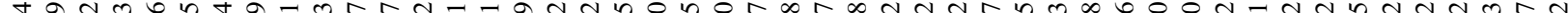

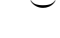

运态

禹空

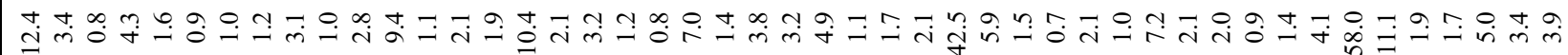

近

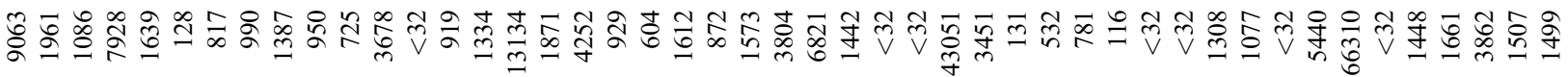

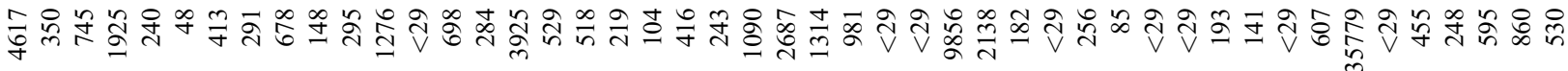

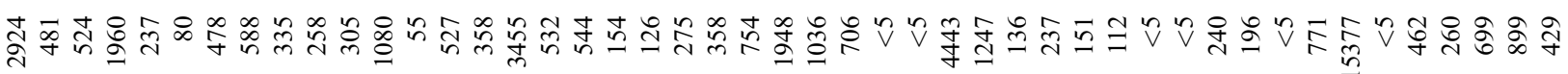

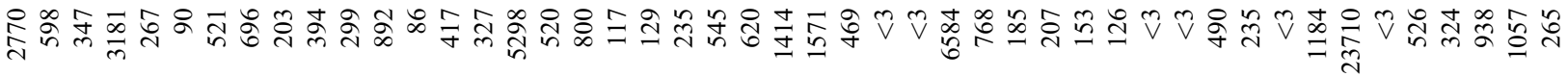

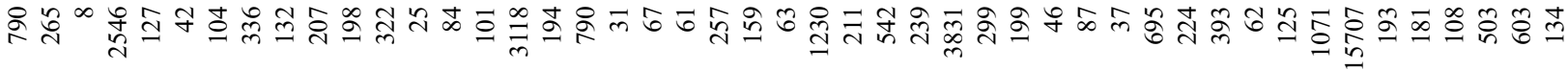

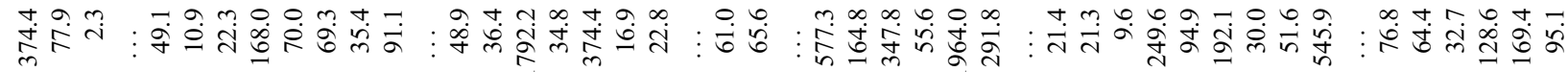

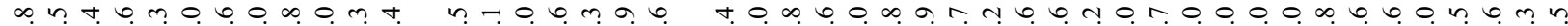

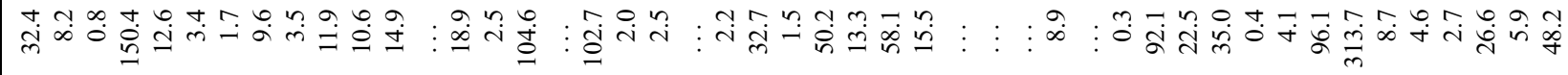

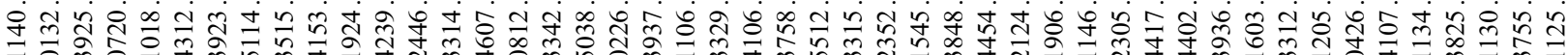

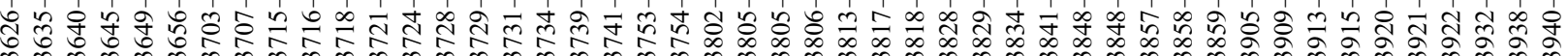

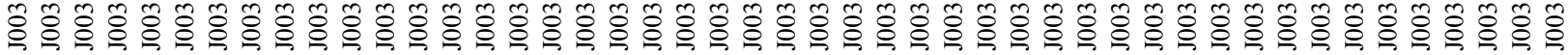

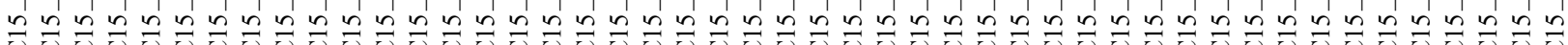

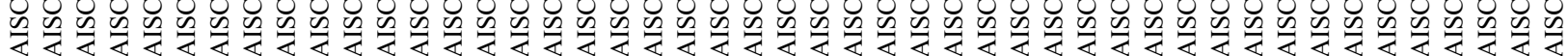

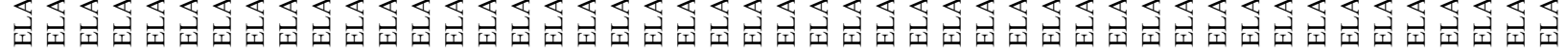




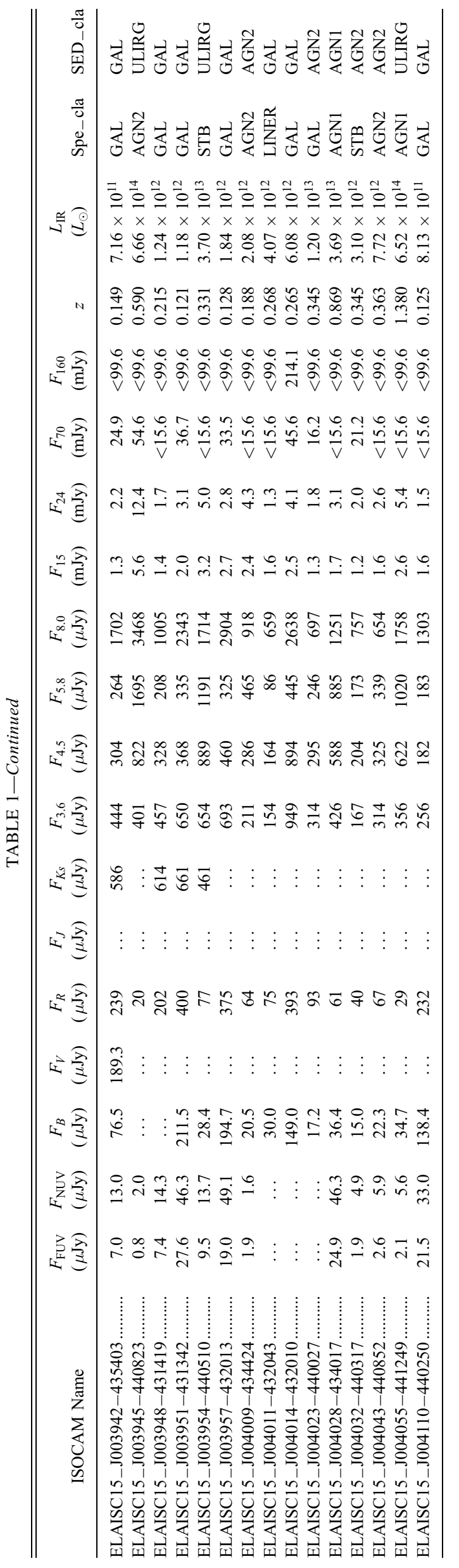




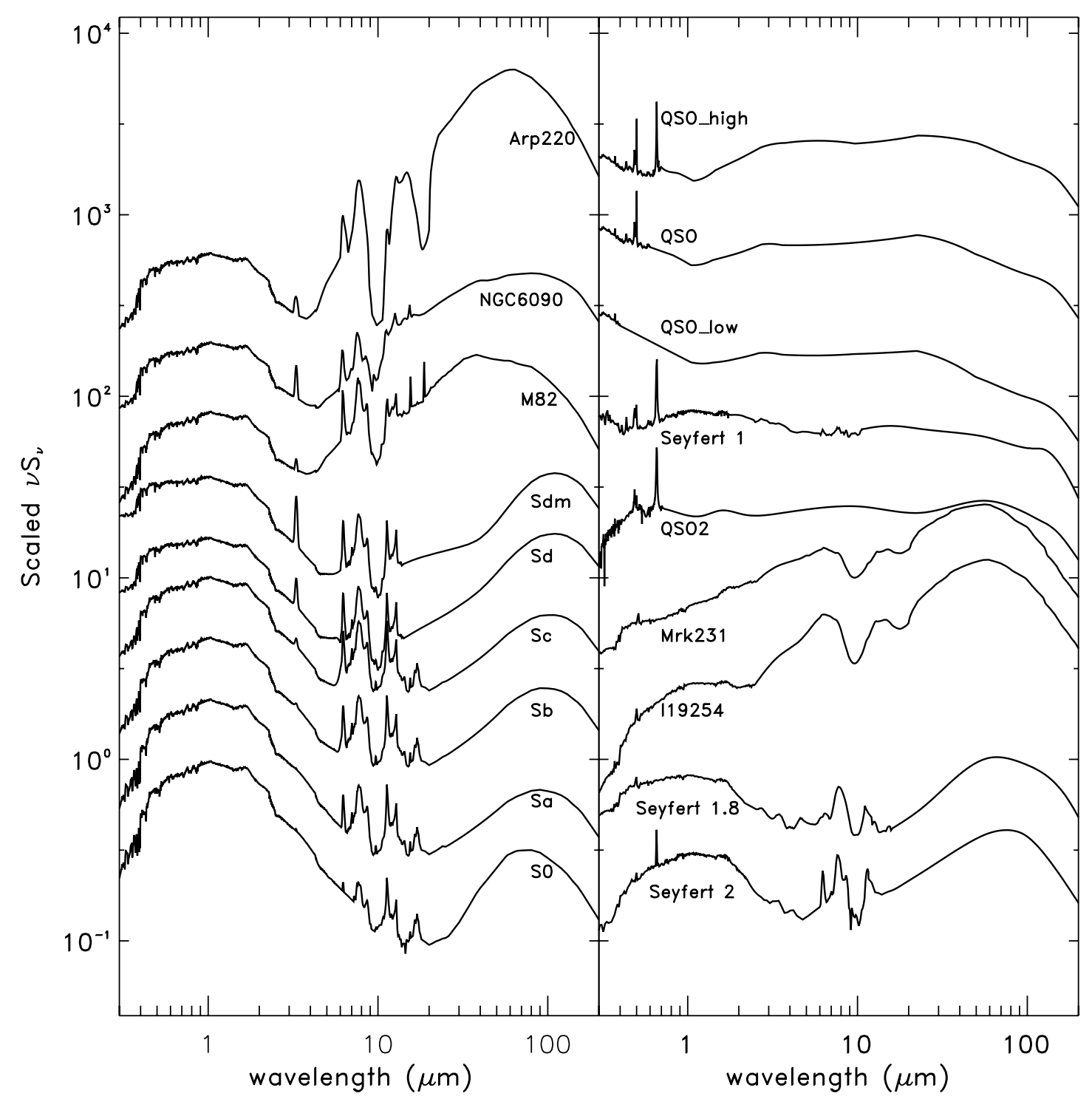

FIG. 1.- Library of template SEDs from Polletta et al. (2007). The SEDs are plotted in arbitrarily scaled luminosity $\nu S_{\nu}$ vs. wavelength.

corresponds to reduced $\chi^{2}$ distributions peaked around 1 (as expected in case of good fit). The enlarged relative errors $\left(\sigma_{\text {object }} /\right.$ $S_{\text {object }}$ ) obtained in this way go, on average, from $\sim 12 \%-15 \%$ in the optical/NIR/IRAC bands up to $\sim 20 \%$ in the MIPS bands.

With the new (significantly larger) photometric uncertainties, which now take into account also the additional uncertainties induced by the template SED discretization, we have run LE PHARE on our sources for the second time, obtaining what we have taken as the final SED-fitting results.

\subsection{The SED-Fitting Results}

In Figure 2 examples of data overlying the best-fitting template SEDs are shown for most of the templates reproducing our data (the original library contains 21 SEDs, but none of our sources could be fitted by any of the 3 elliptical galaxy templates). We find that 83 sources $(41 \%)$ are reproduced by a galaxy template SED (4 S0, $3 \mathrm{Sa}, 16 \mathrm{Sb}, 27 \mathrm{Sc}, 18 \mathrm{Sd}, 15 \mathrm{Sdm}$ ), 13 (6.5\%) with a starburst template SED (1 Arp 220, 5 M82, 7 NGC 6090), 72 (35\%) with a type 2 AGN template SED (52 Seyfert 2, 15 Seyfert 1.8, 5 red QSO), 11 (5.5\%) with an ULIRG composite SED (9 Mrk 231, 2 I19254) and $24(12 \%)$ with a type 1 AGN SED (3 Seyfert 1,6 QSO, 13 high IR/optical flux ratio QSO, 2 low IR/optical flux ratio QSO). Therefore, with our broadband SED-fitting method we find AGN activity in $52.5 \%$ of our MIR-selected sample.

We note that the distributions of the final $\log _{10}\left(S_{\text {object }} / S_{\text {template }}\right)$ values in some bands and for some SED classes are centered on values slightly different from 0 . These offsets, which are different in the different bands and for the different best-fitting SED classes (i.e., for the QSO class the larger offsets are observed at 70 and $160 \mu \mathrm{m}$, while for the galaxy class the larger offsets are observed in the $B, J, K$, and 15 and $24 \mu \mathrm{m}$ bands; see Fig. 3), are interpreted as the average values we should add to the template SEDs to better reproduce the data ensemble of MIR sources. In Figure 3 we show the rest-frame SEDs (dots) of $15 \mu \mathrm{m}$ sources belonging to the different "broad" SED classes (AGN1, ULIRG, AGN2, and starburst and spiral galaxy), compared to the most frequent best-fit template SED of that class (i.e., "QSO" for AGN1, "Seyfert 2" for AGN2, "Mrk 231" for ULIRG, "NGC 6090" for starburst, "Sc" for spiral), all normalized to the $K s$-band flux density. The white triangles represent the average values that the templates should have in the different rest-frame bands to better reproduce the bulk of our sources, while the gray-shaded areas represent their relative uncertainty region corresponding to $\pm 1 \sigma$ of the expected values. From this check we can conclude that, on 


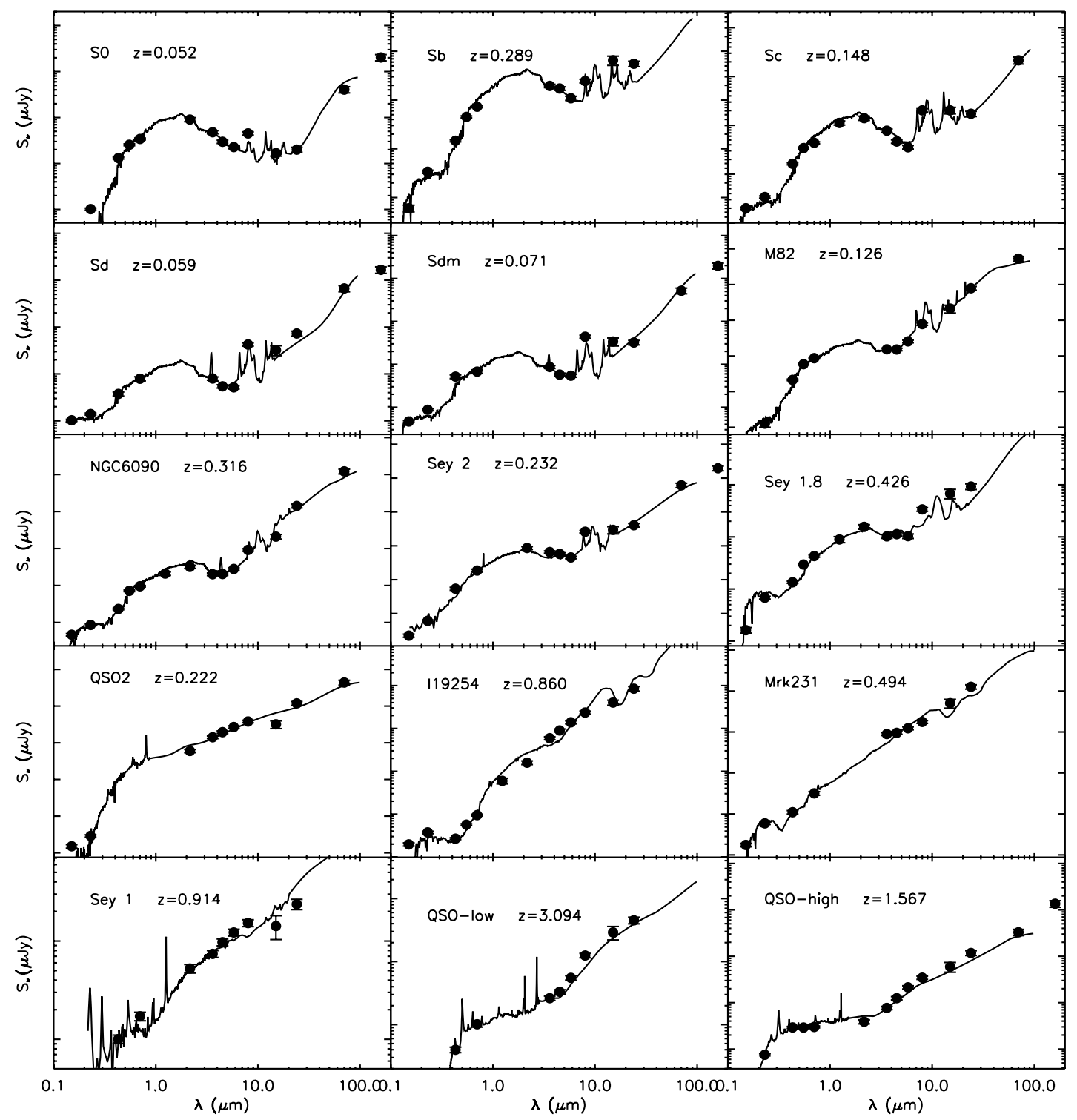

FIG. 2. - Example of SED fits for our sample: the data ( filled circles with error bars), plotted as flux ( $S_{\nu}$ in $\mu \mathrm{Jy}$ ) vs. observed wavelength, are superposed on the corresponding best-fitting template SEDs (solid line), opportunely redshifted at the source's $z$. The plotted uncertainties are those reported in the various catalogs and not those used for the fits.

average, the template SEDs of the Polletta et al. (2007) library modeled on local galaxies and AGNs are able to reproduce also most of the MIR-selected sources at intermediate redshifts, although some of the local templates should be slightly modified in some wavebands to get a better agreement with the bulk of the observed data.

In order to estimate the reliability of the SED classification, we compared the classification and $\chi^{2}$ obtained by the two best solutions of each fit: the primary (corresponding to the minimum $\chi^{2}$ value, $\left.\chi_{\text {best }}^{2}\right)$ and the secondary solutions $\left(\chi_{2 \text { nd }}^{2}\right)$. We find that the primary solutions of our photometric analysis are quite stable within uncertainties. In fact, for the majority of our sources $(92 \%)$ the $\chi_{\text {best }}^{2}$ values are within the $90 \%$ probability threshold $\left(\chi_{90 \%}^{2}=\right.$ 21.07 for the case of 14 degrees of freedom). In most cases the secondary solution belongs to the same "broad" SED class of the primary (i.e., AGN or galaxy, containing all the type 1's, type 2's, and ULIRG templates and all the galaxy and starburst templates, respectively) and only for 23 objects (11\%) it belongs to a different class, with a $\chi_{2 \text { nd }}^{2}$ lower than the $\chi_{90 \%}^{2}$ threshold. In all the other cases, if the primary and secondary solutions correspond to dif- ferent SED classes, the secondary solutions have a low probability of being acceptable $\left(\chi_{2 \text { nd }}^{2}>\chi_{90}^{2} \%\right)$. The 16 sources $(8 \%)$ with a statistically bad primary solution $\left(\chi_{\text {best }}^{2}>\chi_{90 \%}^{2}\right)$ have, in a few cases, some evident photometric errors in one or more bands (mainly in $J$ and $K^{\prime}$ ). In all the remaining cases, the bad fit is due to an observed SED flatter than the best-fitting template in the MIR domain, thus implying the need of a template SED (missing in our library) intermediate between the power-law and the Seyfert 2/1.8 ones, where the AGN is not dominant as in the type 1 template case (power law), but its contribution is higher than in the Seyfert 2 template case.

\section{SPECTROSCOPIC VERSUS SED CLASSIFICATION}

We have compared the results of our SED-fitting procedure with the results obtained from the optical spectroscopy. In Table 2 we show for each spectroscopic class the number of sources which are best fitted by the various SED templates and vice versa. The reported percentages are the fractions of sources of a given spectroscopic classification fitted by the various template SEDs. The 

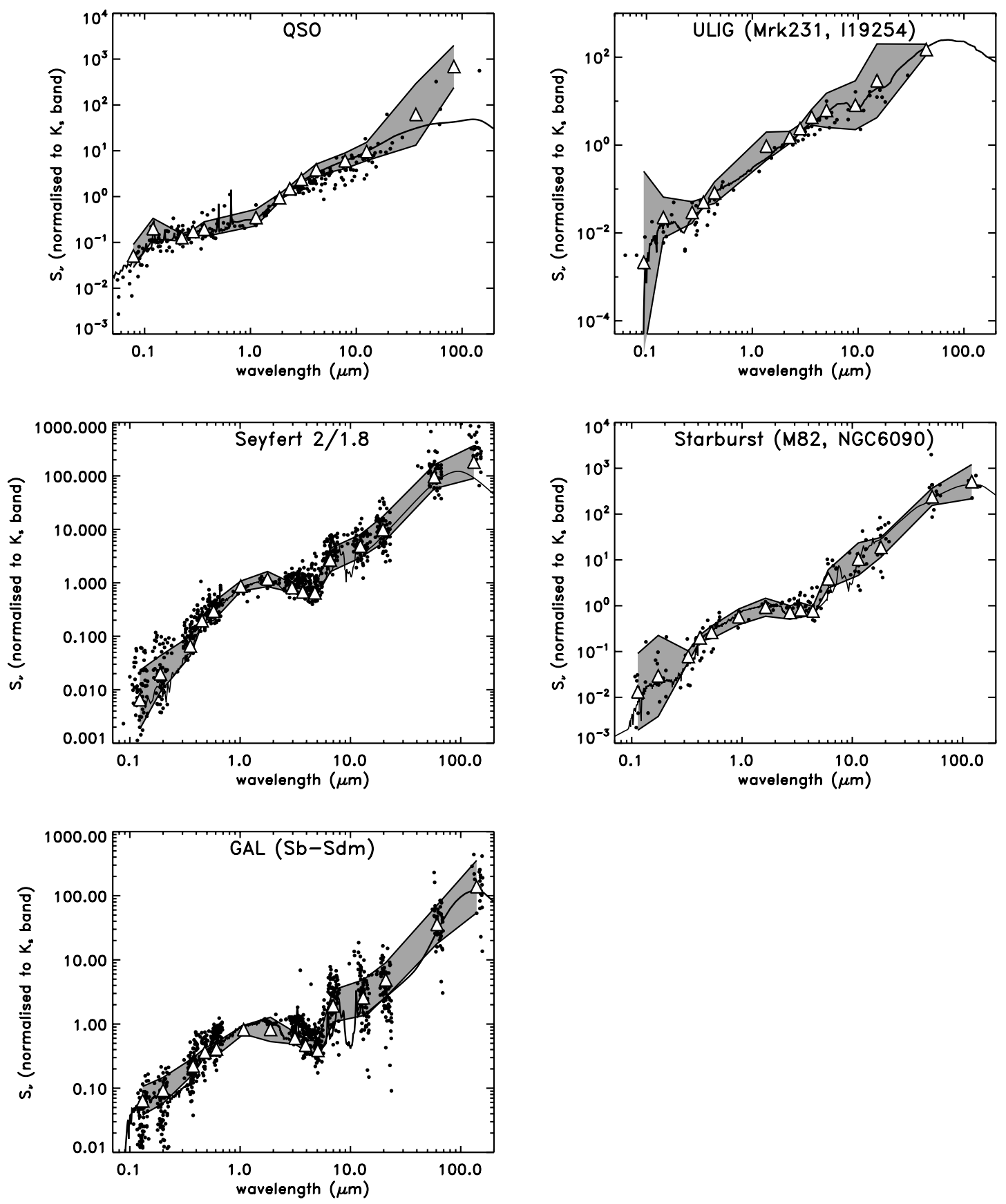

FIG. 3.-Rest-frame SEDs (dots) of $15 \mu \mathrm{m}$ sources belonging to the different broad SED classes (AGN1, ULIRG, AGN2, starburst and spiral galaxy) compared to the most frequent best-fit template SED of that class, all normalized to the $K_{s}$-band flux density. The white triangles represent the average values that the templates should have in the different rest-frame bands to better reproduce our sources, while the gray-shaded areas show their relative uncertainty region corresponding to $\pm 1 \sigma$.

spectroscopic type 1 (broad line) AGNs are all fitted by AGN template SEDs, and therefore the agreement between the two classifications for type 1 AGNs is very good (the only three objects fitted by a type 2 AGN template are indeed fitted by a Seyfert 1.8 template). However, the agreement is not equally good for the spectroscopic type 2 AGNs (+LINERs) and galaxies: $56 \%$ of the AGN2+LINER sample is fitted by a type 2 AGN template and another $19 \%$ is fitted by type $1 \mathrm{AGN}$ or composite (AGN+starburst) template, but $\sim 25 \%$ do show a galaxy SED. Also for the majority $(63 \%)$ of the spectroscopic normal galaxies the two classifications agree (another 3\% is fitted by a starburst template), but there is a significant fraction (34\%) fitted by AGN templates (mostly type 2's). For the spectroscopic starbursts the fraction of sources classified as AGNs by the SED fitting is even higher, $53 \%$. The small number of spectroscopically unclassified sources is fitted for $40 \%$ by AGN and for $60 \%$ by galaxy templates. Therefore, we can conclude that the SED-fitting technique applied to our MIR selected sample is able to identify AGN activity in $~ 40 \%$ of sources spectroscopically unclassified, in $\sim 34 \%$ of sources spectroscopically classified as galaxies, in $~ 53 \%$ of spectroscopic starbursts, in $75 \%$ of type 2 AGNs or LINERs, and in $100 \%$ of type 1 AGNs. The main result of this comparison is that, although for many sources the spectroscopic classification is in agreement with the classification resulting from the SED-fitting method, the broadband SED analysis is able to find AGN activity in a higher fraction of MIR sources $(\sim 53 \%)$ than the optical line diagnostic techniques ( $29 \%)$. On the other hand, a small number (14\%) of the spectroscopically classified AGNs are classified as galaxies by the SED fitting. 
TABLE 2

Spectroscopic Classification versus Sed Classification

\begin{tabular}{|c|c|c|c|c|c|c|}
\hline \multirow[b]{2}{*}{ SED } & & \multicolumn{5}{|c|}{ SpeCtroscopic } \\
\hline & & AGN1 & AGN2+LINER & Starburst & Galaxy & Unclassified \\
\hline Total ……................. & 203 & 27 & $25+7$ & 32 & 107 & 5 \\
\hline 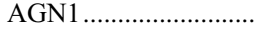 & 24 & $20(74.1 \%)$ & $1+0(3.1 \%)$ & $2(6.2 \%)$ & $1(0.9 \%)$ & 0 \\
\hline 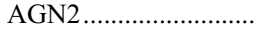 & 72 & $3(11.1 \%)$ & $14+4(56.3 \%)$ & $14(43.8 \%)$ & $35(32.8 \%)$ & $2(40.0 \%)$ \\
\hline $\mathrm{AGN}+$ starburst ............ & 11 & $4(14.8 \%)$ & $5+0(15.6 \%)$ & $1(3.1 \%)$ & $1(0.9 \%)$ & 0 \\
\hline Starburst ......................... & 13 & 0 & $2+1(9.4 \%)$ & $6(18.8 \%)$ & $3(2.8 \%)$ & $1(20.0 \%)$ \\
\hline 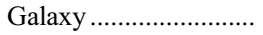 & 83 & 0 & $3+2(15.6 \%)$ & $9(28.1 \%)$ & $67(62.6 \%)$ & $2(40.0 \%)$ \\
\hline
\end{tabular}

For comparison, we cite the recent results of Polletta et al. (2007), who, using a sample of X-ray selected AGNs with available spectroscopic classification and a similar fitting method as applied to our sample, find that $82 \%$ of the X-ray-selected AGNs are classified as AGNs by the SED-based classification method, compared to $78 \%$ by the spectroscopic classification. However, the SED and the spectroscopic classification agree only in 53\% of the cases: the SED method and the spectroscopic classification for X-ray sources are consistent for all type 1 AGNs, but for only $29 \%$ of type 2 AGNs and $33 \%$ of AGNs with galaxy spectra.

The more evident change between the spectroscopic and SED classifications in our sample is due to the fact that several sources optically classified as normal or starburst galaxies do show a Seyfert 2/Seyfert 1.8-like SED, or possibly a composite (starburst+ AGN) SED. Therefore, the total fraction of type 2 AGNs increases from $15.5 \%$ (spectroscopic) to $36 \%$ (SED-fitting), while that of galaxies powered by star formation (normal+starburst) decreases from $70 \%$ (spectroscopic) to $48 \%$ (SED-fitting). In addition, we have $4 \%$ of composite (AGN+starburst) SEDs that were not considered as spectroscopic class.

As a partial explanation of these discrepancies, we note that many of the sources spectroscopically classified as normal galaxies do not have enough lines in their optical spectra to be properly classified according to the standard line ratios diagnostics (i.e., Tresse et al. 1996). In fact, many of them have only $\mathrm{H} \alpha$ and/or [O II] in emission, and therefore the spectroscopic classification might be misleading. However, in all the cases in which the classification changed from spectroscopy to SED-fitting, the spectra do either show no AGN emission lines (e.g., [O III]) at all or ones lower than those due to star formation, allowing them to be clearly classified as galaxy (normal or starburst) through the diagnostics diagrams. In any case, the SED-fitting procedure seems to find more AGNs than the optical spectral lines classification. One possible reason is that in most of these objects the AGN dominates the energetic output only in the MIR. Indeed, most of the sources in our sample with galaxy spectroscopic classification and SEDs reproduced by a Seyfert 2/1.8 template could be fitted by a normal galaxy in the optical/NIR part of their spectra, but not in the MIR (in particular in the $3-8 \mu \mathrm{m}$ range), where the data are too flat to be reproduced by a normal/starburst galaxy SED and therefore require the presence of a low-luminosity AGN. The AGN shows up just in the range where the host galaxy SED has a minimum, due to the junction between the stellar photospheric emission (dominating the optical/NIR) and the dust component (starting to dominate at $\lambda>5 \mu \mathrm{m}$; see, e.g., the late-type spirals and starburst templates in Fig. 1), which is also the range where the hot dust heated by an AGN is expected to start increasing toward the peak. In case an AGN component is present with a NIR luminosity similar to that of the host galaxy, emission from hot dust heated by the AGN contributes to the NIR/MIR filling up the dip observed in star-forming galaxies and producing a flat NIR/MIR spectrum (see, e.g., the Seyfert templates in Fig. 1). This is consistent with the results concerning X-ray-selected obscured AGNs, which are known to be "elusive" in their optical spectra due to host galaxy dilution or heavy dust and gas obscuration or both (see Fiore et al. 2003; Maiolino et al. 2006; Tajer et al. 2007; Caccianiga et al. 2007; Civano et al. 2007; Cocchia et al. 2007). Unfortunately, a check using the X-ray luminosity for our "elusive" AGNs is inconclusive for our purposes, since the XMM-Newton observations cover only a small portion of the field (15\%).

In order to visualize what we discussed above and look at the average properties (in terms of redshift and rest-frame luminosity) of the different SED classes, in Figure 4 we show the redshift (in logarithmic scale) and the $\log \left(L_{15 \mu \mathrm{m}}\right)$ distributions for the different SED classes to which our sources belong. We notice a clear trend of increasing $15 \mu \mathrm{m}$ luminosity (and $z$ ) from early-type (S0, $\mathrm{Sa})$ to later type (Sd, starburst) SEDs. Type 2 AGNs are spread over almost the entire range of $L_{15 \mu \mathrm{m}}\left(\sim 10^{9}-10^{12} L_{\odot}\right.$, except for two $z>2$ objects at $\left.L_{15 \mu \mathrm{m}}>10^{13} L_{\odot}\right)$, and over a large redshift range $(0.05<z<3)$, while type 1 's occupy the region of higher luminosities $\left(L_{15 \mu \mathrm{m}}>10^{10} L_{\odot}\right)$ and redshifts (most of them are at $0.5<z<3$ ). The different spectroscopic classifications have been highlighted by filling the relative distributions with different patterns, as shown in the legend within the plot.

Although very uncertain, since the templates often do not fit well the 70 and $160 \mu \mathrm{m}$ data, the total IR luminosities $\left(L_{\mathrm{IR}}\right)$ have been computed by integrating the best-fitting template SEDs between 8 and $1000 \mu \mathrm{m}$. Most of the starburst galaxies and some of the type 2 AGNs are in the ULIRG luminosity range $\left(L_{\mathrm{IR}}>\right.$ $\left.10^{12} L_{\odot}\right)$, with the remainder in these two classes in the LIRG range $\left(10^{11} L_{\odot}<L_{\mathrm{IR}}<10^{12} L_{\odot}\right)$. The majority of normal galaxies have $L_{\mathrm{IR}}<10^{11} L_{\odot}$, with just some in the LIRG range. All the type 1 AGNs are in the ULIRG range, with a few even in the hyperLIRG (HyLIRG) range $\left(L_{\mathrm{IR}}>10^{13} L_{\odot}\right)$, where all the composite AGNs are instead.

\section{DISCUSSION AND CONCLUSIONS}

We have derived the broadband (FUV to FIR) SEDs for the largest available highly complete (72\%) sample of intermediate- $z$ MIR-selected galaxies and AGNs with spectroscopic identification and redshift. The majority of these objects are at $0.1<$ $z<1.3$ and in the flux density range where strong evolution is observed in the counts of MIR sources. Their broadband SED characterization is therefore fundamental to understanding the nature of sources responsible for the observed evolution. Based on the SED-fitting technique, we have classified the MIR sources, identifying AGN signatures in about $53 \%$ of them. This fraction is significantly higher than that derived from optical spectroscopy ( $\sim 29 \%$ ) and is due principally to the identification of type 2 AGN activity in objects spectroscopically classified as galaxies. This 


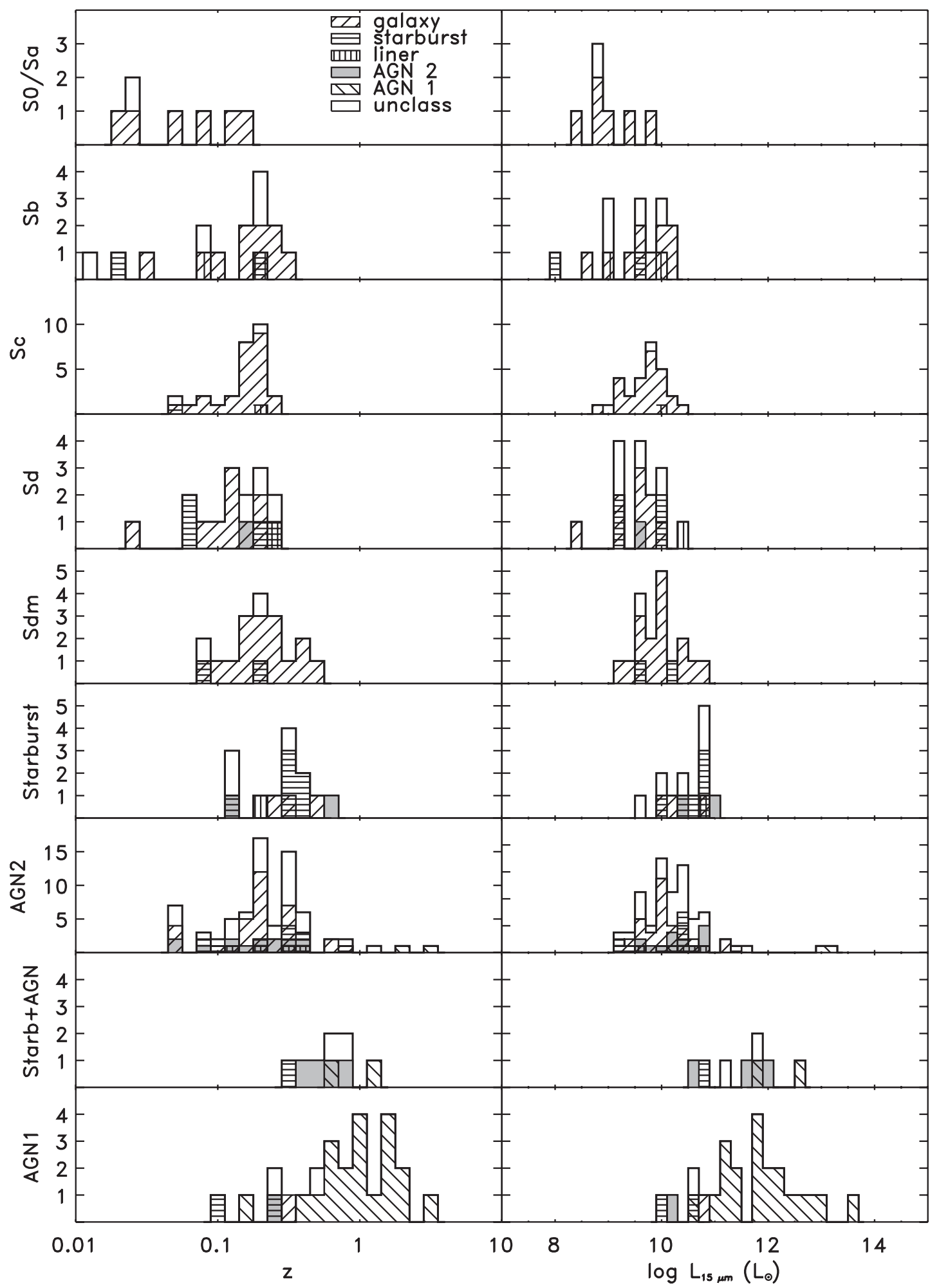

FIG. 4.- Logarithmic distributions of $z$ (left) and $L_{15 \mu \mathrm{m}}$ luminosity (right) for the different SED classes reproducing our data. The luminosities have been derived by considering for each source its best-fitting template SED. Within each distribution the different spectroscopic classes are highlighted, as explained in the legend.

might be caused, at least in part, by host galaxy dilution in the optical, similar to what is observed for obscured AGNs selected in X-rays. It is likely that in most of our objects for which the AGN luminosity in the optical band is fainter than that of the host galaxy, the AGN dominates the energetic output only in the MIR, showing up just in the range where the host galaxy SED has a minimum, while the hot dust heated by the AGN is expected to start peaking.
We note that the fraction of MIR sources with an "elusive" AGN derived in this work is in agreement with recent results from MIR spectroscopy (with Spitzer IRS) of local star-forming galaxies from the Spitzer SINGS sample by Smith et al. (2007), who find that a high fraction ( $~ 50 \%)$ of local galaxies do harbor low-luminosity AGNs (LINER or Seyfert types). These lowluminosity AGNs, in contrast to the luminous ones which completely destroy PAH grains, are able to excite the PAHs and to 
modify the emitting grain distribution, thus providing unusual PAH spectra with either absent $6.2,7.7$, and $8.6 \mu \mathrm{m}$ bands or very weak ones, suppressed in strength by up to a factor of 10. An indication of the presence of such weak AGN systems, which are found to shift power from the short to the long wavelength PAHs, can be revealed, for example, by the strength ratio between the PAH features at 7.7 and at $11.3 \mu \mathrm{m}$ (Smith et al. 2007). Therefore, the definitive confirmation of the presence of weak AGN systems in our intermediate- $z$ MIR dusty galaxies, at present, could be obtained only through IRS spectroscopy, allowing us to test their SED shape indications using line ratios.

Given the new percentages of AGNs, we have updated the relative fractions of AGNs and galaxies contributing to the observed $15 \mu \mathrm{m}$ source counts. Although the results of our work might still be affected, at some level, by photometric errors in the data and by some degrees of degeneracy in the template SEDs, the analysis of the photometric errors and the agreement between the primary and secondary solutions in the vast majority of the cases make us confident that the majority of the SED-classified AGNs are reliable.

For coherence with the previous classification and source counts (see La Franca et al. 2004 and considerations therein) and to avoid biasing toward AGNs, we have treated all the sources without spectroscopic redshift as galaxies. In fact, a spectroscopic sample $72 \%$ complete can be biased toward AGNs, since the large majority of the spectroscopically unidentified objects have $15 \mu \mathrm{m}$ flux densities fainter than $1 \mathrm{mJy}$. Galaxies dominate over AGNs in this range of flux, and it is therefore likely that most of the unidentified objects are galaxies.

On the other hand, since we know that optical spectroscopy can miss AGN signatures, we can take the fraction of spectroscopic AGNs (used for deriving the AGN LF at $15 \mu \mathrm{m}$ by Matute et al. 2006) as a lower limit. In Figure 5 we show the fractions of AGNs as function of $15 \mu \mathrm{m}$ flux obtained by considering the spectroscopic classification (dashed line; from Matute et al. 2006) and the SED classification ( filled circles with error bars; this work). Other recent works (Brand et al. 2006; Treister et al. 2006), although based on different diagnostics (e.g., the 24 to $8 \mu$ m flux ratio or X-ray detection), estimate lower limits to the AGN contribution in MIR surveys significantly higher than those predicted by Matute et al. (2006) and in very good agreement with the fractions derived in this paper (see Fig. 5, where the Brand et al. 2006 and Treister et al. 2006 results are reported for comparison, with the $24 \mu \mathrm{m}$ flux densities converted to $15 \mu \mathrm{m}$ by using the opportune $24 / 15 \mu \mathrm{m}$ ratio as function of flux as computed by Gruppioni et al. 2005). Such results might have a significant impact on the interpretation of galaxy and AGN evolution and on the physics of the MIR-selected objects, so far often erroneously assumed in many models to be all starburst galaxies. In fact, all the existing models of galaxy and AGN evolution in the IR should be revised by taking into account the higher fraction of AGNs dominating in the MIR wave range.

By comparing the AGN fraction derived by our SED-fitting analysis to the "lower limit" coming from the spectroscopic classification, we can determine how the relative source counts of galaxies and AGNs (and consequently the relative evolutionary models) should change according to the new values. For this purpose, in Figure 6 we have plotted the extragalactic source counts at $15 \mu \mathrm{m}$ in the $\mathrm{S} 1$ field (Gruppioni et al. 2002) with the relative contribution of galaxies and AGNs computed according to the previous (left) and new (right) determination of AGN fractions. The difference between the two is small at bright fluxes, but the SED-fitting method finds more and more AGNs going to fainter fluxes. The galaxy population now dominates below $\sim 3 \mathrm{mJy}$

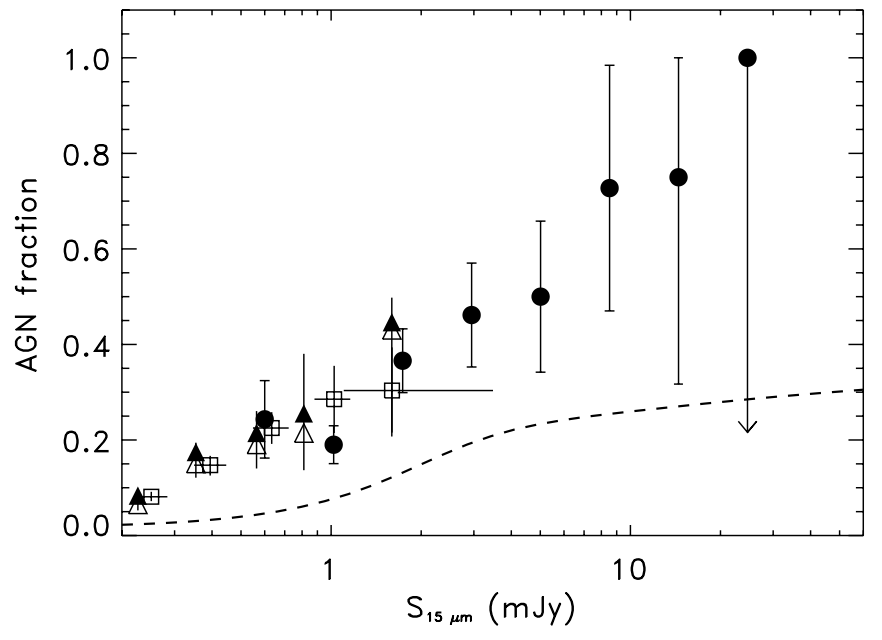

FIG. 5.- Fraction of AGN over total extragalactic sources as a function of the $15 \mu \mathrm{m}$ flux density, as derived from the spectroscopic classification (dashed line; Matute et al. 2006) and from the SED-fitting performed in this work ( filled circles with error bars). For comparison, the results of Brand et al. (2006) and Treister et al. (2006) have been plotted as open squares and triangles (open triangles: uncorrected for AGNs not detected in X-rays; filled triangles: corrected for AGNs not detected in X-rays), respectively.

(previously 6-7 mJy), although now the AGN counts are only a factor of 2 lower even at the lower flux densities. For comparison, we have also shown the results of a recent work by La Franca et al. (2007), who updated the AGN source counts at $15 \mu \mathrm{m}$ on the basis of X-ray-band (XMM-Newton) observations (Puccetti et al. 2006) on the central $0.6 \mathrm{deg}^{2}$ of ELAIS-S1. By classifying as AGNs all the MIR sources with an unabsorbed $2-10 \mathrm{keV}$ X-ray luminosity $>10^{42} \mathrm{ergs} \mathrm{s}^{-1}$, the authors find that at least $13 \%$ of the galaxies previously classified on optical basis (by La Franca et al. 2004) do harbor an AGN, therefore concluding that $>24 \%$ of the $15 \mu \mathrm{m}$ sources with flux density $>0.6 \mathrm{mJy}$ are AGNs. The AGN source counts at $15 \mu \mathrm{m}$ updated by La Franca et al. (2007) and plotted in Figure 6 (dot-dot-dashed line) are indeed significantly higher than those based on optical classification, and are in better agreement with (although somewhat lower than) the AGN counts derived in this work. The higher efficiency of the SED-fitting technique with respect to X-ray luminosities in detecting AGN activity, in this specific case, might be partially due to the fact that $X M M$ Newton observations in $\mathrm{S} 1$ are not deep enough $[S(2-10 \mathrm{keV})=$ $2 \times 10^{-15} \mathrm{ergs} \mathrm{cm}^{-2} \mathrm{~s}^{-1}$ ] to detect (and thus allow conclusive results for) all (or most) of our sources. Moreover, it is known that even the deepest X-ray surveys fail in detecting highly obscured (i.e., Compton thick: $N_{\mathrm{H}}>10^{24} \mathrm{~cm}^{-2}$ ) AGNs, while sensitive measurements in the IR range, and in particular SED studies in the NIR/MIR range, provide a unique and efficient opportunity to recover the fraction of obscured or "elusive" AGNs not identified in X-ray surveys (Alonso-Herrero et al. 2006; Fiore et al. 2008; Martinez-Sansigre \& Rawlings 2007; Daddi et al. 2007).

We have compared our results to those of Hickox et al. (2007) although the sample selected by those authors is an IRAC-selected one which also fulfills additional selection criteria (i.e., detection in all the IRAC bands and in the $R$ band, IRAC colors falling in the Stern et al. 2005 AGN-selection region, spec- or photo$z>0.7$ ) - with the finding that according to the Hickox et al. criteria, just a few of our AGNs can be considered "obscured." In fact, very few of our AGNs fall below the $R-[4.5]=6.1$ separation boundary considered by Hickox et al. (2007) to divide obscured from unobscured AGNs. All our type 1's and most of our type 2's are below that boundary, while most of the composite AGNs 


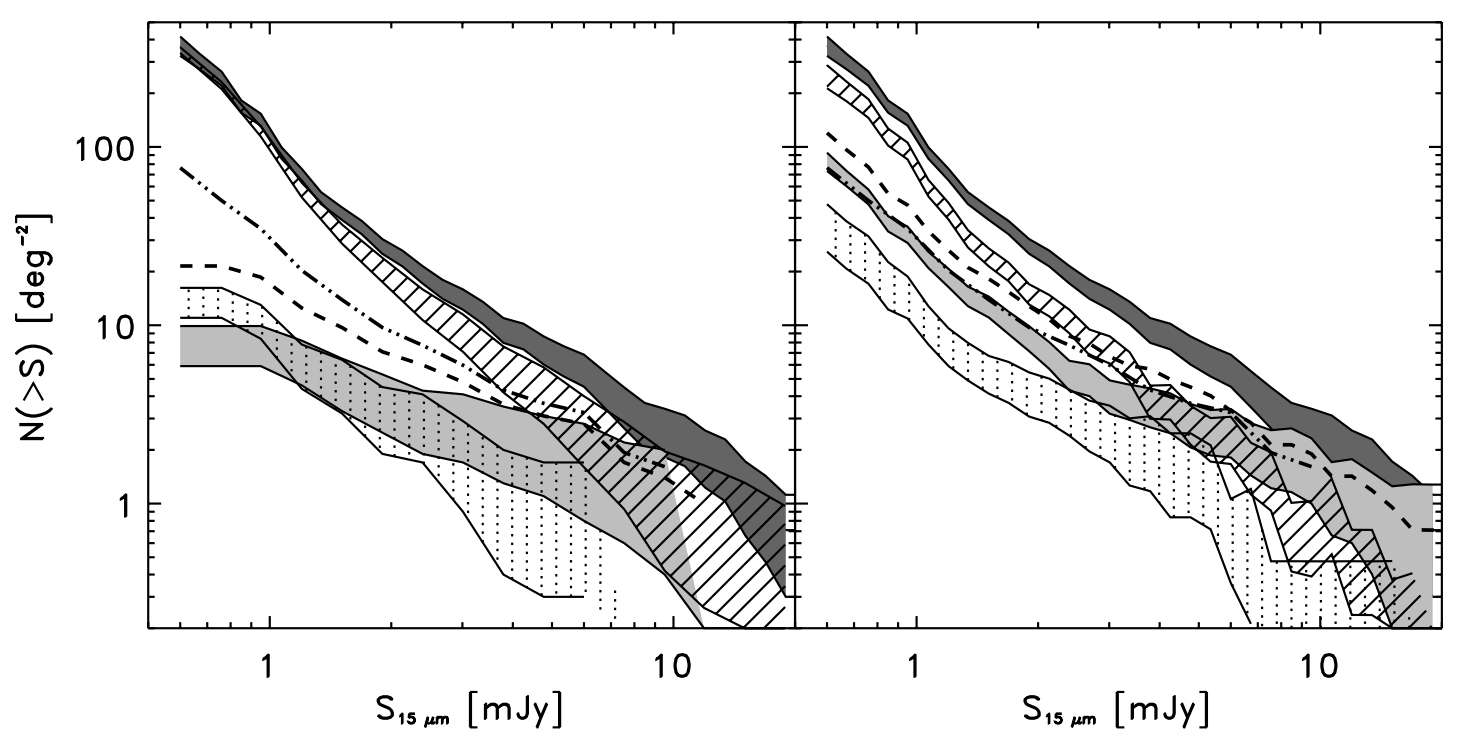

FIG. 6.-Observed extragalactic source counts at $15 \mu \mathrm{m}$ in ELAIS-S1: total counts with uncertainties (dark gray shaded area); type 2 AGN contribution (light gray shaded area); type 1 AGN contribution (dotted area); galaxy contribution (hatched area). The total (type 1+type 2) AGN contribution is shown by the dashed line, while the dot-dot-dashed line represents the total AGN counts at $15 \mu \mathrm{m}$ updated by La Franca et al. (2007) on the basis of hard X-ray observations. In the left panel the AGN/galaxy contributions are derived from the spectroscopic classification (La Franca et al. 2004), while in the right panel they come from the SED-fitting work described in this paper. All the optically unidentified sources have been conservatively considered to be galaxies in both plots (see $\S 5$ ).

are above. This would imply that most of our composite AGNs are obscured and all our type 1 and most of our type 2 AGNs are unobscured. The cumulative redshift distribution of our $z>0.7$ AGNs is in good agreement with that derived by Hickox et al. (2007), up to $z=1.2-1.3$. Above $z=1.3$ there are just few sources in our sample, due to our relatively bright $15 \mu \mathrm{m}$ and $R$-band selection, therefore we can consider our AGN (in particular type 2) sample complete only up to $z=1.3$.

The results of this work will be very useful for updating all the models aimed at interpreting the deep infrared survey data and, in particular, for constraining the nature and the role of dustobscured systems in the intermediate/high-redshift universe. The main changes to the actual evolutionary models for IR galaxies and AGNs would consist in better defined evolutionary source classes (and SEDs) and in updated evolutions for the different classes. In particular, in reference to the specific case of the Pozzi et al. (2004) and Matute et al. (2006) models, which are based on the same sample considered here, although star-forming galaxies are still the dominant class at low flux densities, their evolution should be lower than that derived by Pozzi et al. (2004). Similarly, the AGNs (mainly type 2's) should evolve more rapidly (and probably both in luminosity and density) than derived by Matute et al. (2006), similar to star-forming galaxies. In fact, the higher fraction of AGNs in infrared surveys than that found through optical spectroscopy is a result common to various works (i.e., Brand et al. 2006; Treister et al. 2006) and applies mainly to type 2 AGNs. Type 1 AGNs are in fact easier to identify in the optical, due to their broad lines, and thus most (or all) of them are classified from their optical spectra. Type 2 AGNs are more "elusive" and difficult to reveal in the optical. Therefore, the higher fraction of IR AGNs is mainly due to the unveiling of type 2 AGNs previously erroneously classified as galaxies. For this reason, the starburst galaxies, to which all the evolution observed in the MIR was commonly attributed, probably exist in smaller numbers than formerly believed, and part of their evolution should be ascribed to type 2 AGNs. This is also evident from the right panel of Figure 6, where the revised AGN source counts increase toward fainter fluxes with a slope similar to (although slightly lower than) that of galaxies, while, according with the results based on op- tical spectroscopy (left panel), the AGNs were rapidly converging toward the lower flux densities.

Finally, instead of considering nonevolving normal galaxies (and therefore ascribing all the evolution to starburst galaxies), it would be more appropriate to consider galaxy SEDs evolving with $z$ (and/or $L$ ) from early- to late-type (and to starburst), as suggested by the results shown in Figure 4, eventually taking into account different evolutions for different luminosity intervals. The revision of evolutionary models for IR sources based on the results presented here is beyond the aims of this work and will be treated in a forthcoming paper (C. Gruppioni et al. 2008, in preparation).

To summarize, through the broadband SED analysis of the largest available highly complete (72\%) spectroscopic sample of MIR-selected galaxies and AGNs at intermediate $z$, in this work we have

1. verified the assumption that local template SEDs are also able to reproduce most of the MIR-selected galaxies and AGNs at $0.1<z<1.3$;

2. found AGN activity in a significantly higher fraction of sources ( $\sim 53 \%)$ than derived from optical spectroscopy ( $29 \%)$;

3 . derived new relative fractions of AGNs and galaxies contributing to the observed MIR source counts, with AGNs going from $\sim 10 \%$ to $20 \%$ of the MIR population at $S_{15}<0.6 \mathrm{mJy}$ up to $\sim 90 \%-100 \%$ at $S_{15}>10 \mathrm{mJy}$;

4. computed new source counts in the MIR, with the AGN counts (especially type 2's) now increasing with a slope similar to that of galaxies. This result is likely to imply different evolutionary rates than formerly considered by the present models (i.e., lower rates for starburst galaxies and higher rates for type 2 AGNs).

The authors acknowledge financial contribution from the contracts PRIN-INAF 1.06.09.05, ASI-INAF I/023/05/0, and PRINMIUR 2006025203. We thank F. Fiore and M. Mignoli for useful discussions. We are grateful to the anonymous referee for useful comments that improved the quality of the paper. 
Alexander, D. M., et al. 2001, ApJ, 554, 18

Alonso-Herrero, A., et al. 2006, ApJ, 640, 167

Berta, S. 2005, Ph.D. thesis, Univ. Padua

Berta, S., Fritz, J., Franceschini, A., Bressan, A., \& Pernechele, C. 2003, A\&A, 403, 119

Berta, S., et al. 2006, A\&A, 451, 881

Brand, K., et al. 2006, ApJ, 644, 143

Bruzual, A. G., \& Charlot, S. 2003, MNRAS, 344, 1000

Burgarella, D., et al. 2005, ApJ, 619, L63

Caccianiga, A., Severgnini, P., Della Ceca, R., Maccacaro, T., Carrera, F. J., \& Page, M. J., 2007, A\&A, 470, 557

Cesarsky, C. J., et al. 1996, A\&A, 315, L32

Chary, R., \& Elbaz, D. 2001, ApJ, 556, 562

Chary, R., et al. 2004, ApJS, 154, 80

Civano, F., et al. 2007, A\&A, 476, 1223

Cocchia, F., et al. 2007, A\&A, 466, 31

Coleman, G. D., Wu, C.-C., \& Weedman, D. W. 1980, ApJS, 43, 393

Daddi, E., et al. 2007, ApJ, 670, 173

Devriendt, J. E. G., Guiderdoni, B., \& Sadat, R. 1999, A\&A, 350, 381

Dias, J. E. 2008, A\&A, submitted

Elbaz, D., et al. 1999, A\&A, 351, L37

Farrah, D., Afonso, J., Efstathiou, A., Rowan-Robinson, M., Fox, M., \& Clements, D. 2003, MNRAS, 343, 585

Fioc, M., \& Rocca-Volmerange, B. 1997, A\&A, 326, 950

Fiore, F., et al. 2003, A\&A, 409, 79 2008, ApJ, 672, 94

Franceschini, A., et al. 2001, A\&A, 378, 1 2005, AJ, 129, 2074

Genzel, R., Lutz, D., \& Tacconi, L. 1998, Nature, 395, 859

Gruppioni, C., Pozzi, F., Lari, C., Oliver, S., \& Rodighiero, G. 2005, ApJ, 618, L9

Gruppioni, C., et al. 1999, MNRAS, 338, 555 2002, MNRAS, 335, 831

Hacking, P. B., Houck, J. R., \& Condon, J. J. 1987, ApJ, 316, L15

Hatziminaoglou, E., et al. 2005, AJ, 129, 1198

Hickox, R. C., et al. 2007, ApJ, 671, 1365

Hook, I. M., Becker, R. H., McMahon, R. G., \& White, R. L. 1998, MNRAS, 297,1115

Ilbert, O., et al. 2006, A\&A, 457, 841

Jarrett, T. H., Chester, T., Cutri, R., Schneider, S., Skrutskie, M., \& Huchra, J. P. 2000, AJ, 119, 2498

Jarrett, T. H., et al. 2006, AJ, 131, 261

Kessler M. F., et al. 1996, A\&A, 315, L27

La Franca, F., et al. 2004, AJ, 127, 3075 2007, A\&A, 472, 797

Lagache, G., Dole, H., \& Puget, J.-L. 2003, MNRAS, 338, 555

Lagache, G., et al. 2004, ApJS, 154, 112

Lari, C., et al. 2001, MNRAS, 325, 1173
REFERENCES

Le Floc'h, E., et al. 2005, ApJ, 632, 169

Lonsdale, C. J., Farrah, D., \& Smith, H. E. 2006, in Astrophysics Update 2, ed.

J. W. Mason (Heidelberg: Springer), 285

Lonsdale, C. J., \& Hacking, P. B. 1989, ApJ, 339, 712

Lonsdale, C. J., et al. 2003, PASP, 115, 897 2004, ApJS, 154, 54

Maddox, S. J., Efstathiou, G., Sutherland, W. J., \& Loveday, J. 1990, MNRAS, 243, 692

Maiolino, R., et al. 2006, A\&A, 445, 457

Marleau, F. R., Fadda, D., Appleton, P. N., Noriega-Crespo, A., Im, M., \& Clancy, D. 2007, ApJ, 663, 218

Marleau, F. R., et al. 2004, ApJS, 154, 66

Martin, D. C., et al. 2005, ApJ, 619, L1

Martinez-Sansigre, A., \& Rawlings, S. 2007, in ASP Conf. Ser. 373, The Central Engine of Active Galactic Nuclei, ed. L. C. Ho \& J.-M. Wang (San Francisco: ASP), 728

Matute, I., LaFranca, F., Pozzi, F., Gruppioni, C., Lari, C., \& Zamorani, G. 2006, A\&A, 451, 443

Matute, I., et al. 2002, MNRAS, 332, L11

Middleberg, E., et al. 2008, AJ, in press

Oliver, S., et al. 2000, MNRAS, 316, 749

Papovich, C., et al. 2004, ApJS, 154, 70

Pearson C. 2005, MNRAS, 358, 1417

Pèrez-Gonzàlez, P. G., et al. 2005, ApJ, 630, 82

Polletta, M., et al. 2006, ApJ, 642, 673 2007, ApJ, 663, 81

Pozzi, F., et al. 2004, ApJ, 609, 122

Puccetti, S., et al. 2006, A\&A, 457, 501

Rowan-Robinson, M. 2001, ApJ, 549, 745

Rowan-Robinson, M., et al. 2004, MNRAS, 351, 1290

Sajina, A., et al. 2007, MNRAS, 369, 939

Sanders, D. B., \& Mirabel, I. F. 1996, ARA\&A, 34, 749

Saunders, W., Rowan-Robinson, M., Lawrence, A., Efstathiou, G., Kaiser, N., Ellis, R. S., \& Frenk, C. S. 1990, MNRAS, 242, 318

Silva, L., Granato, G. L., Bressan, A., \& Danese, L. 1998, ApJ, 509, 103

Smith, J. D. T., et al. 2007, ApJ, 656, 770

Stern, D., et al. 2005, ApJ, 631, 163

Surace, J. A., et al. 2005, BAAS, 37, 1246

Tajer, M., et al. 2007, A\&A, 467, 73

Treister, E., et al., 2006, ApJ, 640, 603

Tresse, L., Rola, C., Hammer, F., Stasińska, G., Le Fevre, O., Lily, S. J., \& Crampton, D. 1996, MNRAS, 281, 847

Vanzella, E., et al. 2005, A\&A, 434, 53 2006, A\&A, 454, 423

Weedman, D., et al. 2006, ApJ, 653, 101

Werner, M. W., et al. 2004, ApJS, 154, 1

Xu, C. K., Lonsdale, C. J., Shupe, D. L., Franceschini, A., Martin, C., \& Schiminovich, D. 2003, ApJ, 587, 90 